\title{
OPTIMAL EXTENSIONS FOR POSITIVE ORDER CONTINUOUS OPERATORS ON BANACH FUNCTION SPACES
}

\author{
O. DELGADO \\ Departamento de Matemática Aplicada I, E. T. S. de Ingeniería de Edificación, \\ Universidad de Sevilla, Avenida Reina Mercedes, \\ 4 A, 41012 Sevilla, Spain \\ e-mail: olvido@us.es
}

(Received 27 November 2012; accepted 4 February 2013; first published online 13 August 2013)

\begin{abstract}
In this paper we give conditions under which a positive order continuous operator $T$ defined on a Banach function space can be extended, preserving the order continuity in a certain optimal way. The optimal domain for $T$ turns out to be a space of weakly integrable functions with respect to a vector measure (defined on a $\delta$-ring) canonically associated to $T$. A similar result is obtained when $T$ is $\sigma$-order continuous and we want to preserve the $\sigma$-order continuity. We apply these results to kernel operators.
\end{abstract}

2010 Mathematics Subject Classification. Primary 46G10; Secondary 46E30, 46B42.

1. Introduction. Let $X(\mu)$ be a Banach function space (briefly, B.f.s.) related to some measure space $(\Omega, \Sigma, \mu)$, and $T: X(\mu) \rightarrow E$, a linear operator satisfying a certain property with values in a Banach space $E$. An interesting question is as follows: Is it possible to extend $T$ to a larger domain preserving the same property and the values in $E$, and in this case, is the extension optimal in some sense. This problem has been studied attending to the continuity property for classical operators in numerous works as in, for instance, $[5,7,9,16,17]$. For a general continuous operator $T$, if $X(\mu)$ is order continuous and $L^{\infty}(\mu) \subset X(\mu)$, we can consider the vector measure $v_{T}: \Sigma \rightarrow E$ given by $v_{T}(A)=T\left(\chi_{A}\right)$. Then the space $L^{1}\left(v_{T}\right)$ of integrable functions with respect to $v_{T}$ is the largest order continuous B.f.s. to which $T$ can be extended as a continuous operator still with values in $E$. This result was proved in [6] for $\mu$ finite and $X(\mu)$ being a B.f.s. in the sense of Lindenstrauss and Tzafriri [12], but the proof works in our case. The condition $L^{\infty}(\mu) \subset X(\mu)$, which is crucial for defining $v_{T}$ on $\Sigma$, excludes important spaces as $L^{1}[0, \infty)$. However, we can forget about this condition if we consider $v_{T}$ defined in an appropriate $\delta$-ring instead of $\Sigma$. This was done in [4], where a similar result is obtained for continuous operators. Another type of property for $T$ involving norms, which includes a certain inequality coming from the Pisier's factorization theorem through weighted Lorentz spaces, is also studied in [4]. It is proved that $T$ can be optimally extended (preserving this type of property) to a certain subspace of $L^{1}\left(v_{T}\right)$, which can be described as a space of multiplication operators.

In this paper, we are interested in properties for $T$ related to the order, namely order continuity and $\sigma$-order continuity. We deal with positive operators with values in 
a Banach lattice and look for optimal extensions preserving these order properties. The key will be again the integration with respect to $v_{T}$, but now further extensions to larger spaces than $L^{1}\left(v_{T}\right)$ are obtained. More precisely, associated to the vector measure $v_{T}$ there are two other important spaces, the space $L_{w}^{1}\left(v_{T}\right)$ of weakly integrable functions and the $\sigma$-Fatou completion $\left[L^{1}\left(v_{T}\right)\right]_{\sigma-\mathrm{F}}$ of $L^{1}\left(v_{T}\right)$, for which $L^{1}\left(v_{T}\right) \subset\left[L^{1}\left(v_{T}\right)\right]_{\sigma-\mathrm{F}} \subset$ $L_{w}^{1}\left(v_{T}\right)$. Taking account that under appropriate conditions the integration operator $I_{v_{T}}$ extends $T$ to $L^{1}\left(v_{T}\right)$, the idea is to extend $I_{\nu_{T}}$ to these larger spaces, and this can be done by using the order properties of $T$.

The paper is organized as follows. Some preliminaries on Banach lattices, Banach functions spaces and integration with respect to a vector measure are given in Section 2. In Section 3 we present some results, which will be used in the proofs of our main Theorems 4.1 and 5.1. In particular, we give conditions under which the integration operator of a general vector measure $v$ can be extended to $L_{w}^{1}\left(v_{T}\right)$ or $\left[L^{1}\left(v_{T}\right)\right]_{\sigma-\mathrm{F}}$ (Propositions 2.7 and 2.8). Fatou-type properties will play an important role in our proofs. In Section 4 we will see that, whenever the order continuous part $X(\mu)_{a}$ of $X(\mu)$ is super order dense in $X(\mu)$, the operator $T$ can be optimally extended preserving the $\sigma$-order continuity to the space $\left[L^{1}\left(v_{T a}\right)\right]_{\sigma-\mathrm{F}}$, where $T a$ is the restriction of $T$ to $X(\mu)_{a}$ (Theorem 4.1). If we want to preserve the order continuity of $T$, then we need that $X(\mu)_{a}$ is order dense in $L^{0}(\mu)$, in which case the optimal domain for $T$ is $L_{w}^{1}\left(v_{T a}\right)$ (Theorem 5.1). Although the line of the proof of this result is the same as in Theorem 4.1, since the jump from sequences to nets is always delicate, we prefer to write it with detail in Section 5. Along the paper, we will consider two natural cases for which our main theorems can be rewritten in a simpler way: the $\sigma$-finite case (i.e. $X(\mu)_{a}$ has a weak unit) and the discrete case (i.e. $\Sigma=2^{\Omega}$ and $\chi_{\{\omega\}} \in X(\mu)$ with $T\left(\chi_{\{\omega\}}\right) \neq 0$ for all $\left.\omega \in \Omega\right)$. Finally in Section 6 we apply our results to kernel operators.

\section{Preliminaries.}

2.1. Banach lattices. In general, we follow the notation and terminology of [13] and [18]. Let $E$ be a real Banach lattice with norm $\|\cdot\|$ and order $\leq$. A closed subspace $F$ of $E$ is an ideal of $E$ if $|x| \leq|y|$ with $x \in E$, and $y \in F$ implies that $x \in F$. An ideal $F$ is order dense in $E$ if for every $0 \leq x \in E$ there exists an upwards directed system $\left(x_{\tau}\right) \subset F$ such that $0 \leq x_{\tau} \uparrow x$. If for every $0 \leq x \in E$ there exists an increasing sequence $\left(x_{n}\right) \subset F$ such that $0 \leq x_{n} \uparrow x$ then $F$ is said to be super order dense in $E$. A weak unit of $E$ is an element $0 \leq e \in E$ such that $x \wedge e=0$ implies $x=0$. If every non-empty subset which is bounded from above has a supremum, then $E$ is said to be Dedekind complete. If the same holds but only for countable subsets then $E$ is called Dedekind $\sigma$-complete. We say that $E$ has the Fatou property if for every $\left(x_{\tau}\right) \subset E$ upwards directed system $0 \leq x_{\tau} \uparrow$ such that sup $\left\|x_{\tau}\right\|<\infty$, it follows that there exists $x=\sup x_{\tau}$ in $E$ and $\|x\|=\sup \left\|x_{\tau}\right\|$, and $E$ has the $\sigma$-Fatou property if the same holds but for increasing sequences. Note that it follows from [13, Theorem 23.2] that if $E$ has the Fatou (or $\sigma$-Fatou) property then it is Dedekind complete (or $\sigma$-complete). The Banach lattice $E$ is order continuous if for every $\left(x_{\tau}\right) \subset E$ downwards directed system $x_{\tau} \downarrow 0$ it follows $\left\|x_{\tau}\right\| \downarrow 0$. When the same holds for decreasing sequences then $E$ is called $\sigma$-order continuous. We denote by $E_{\text {an }}$ the order continuous part of $E$, that is, the 
largest order continuous ideal in $E$. It can be described as

$$
E_{a n}=\left\{x \in E:|x| \geq x_{\tau} \downarrow 0 \text { implies }\left\|x_{\tau}\right\| \downarrow 0\right\},
$$

see [18, Theorem 102.8]. The $\sigma$-order continuous part of $E$, denoted by $E_{a}$, can be described in a similar way by taking decreasing sequences. Of course, $E_{a n} \subset E_{a}$. In the case where $E_{a}$ is Dedekind $\sigma$-complete, it follows that $E_{a n}=E_{a}$, see [18, Theorem 103.6].

Let $T: E \rightarrow F$ be a linear operator between Banach lattices. The operator $T$ is positive if $T e \geq 0$ for every $0 \leq e \in E$, in which case it follows that $T$ is continuous, see [18, Theorem 83.12]. If $T$ is positive and $T e_{\tau} \uparrow T e$ in $F$ whenever $0 \leq e_{\tau} \uparrow e$ in $E$ then $T$ is said to be order continuous ( $\sigma$-order continuous for sequences). We will say that $T$ is $\sigma$-order $w$-continuous if whenever $0 \leq e_{n} \uparrow e$ in $E$ we have $T e_{n} \rightarrow T e$ weakly in $F$ (valid for $F$ being only a Banach space). This last notion comes from [6, Theorem 3.1].

LEMMA 2.1. The following statements hold:

(a) If $T$ is continuous and $E$ is $\sigma$-order continuous, then $T$ is $\sigma$-order $w$-continuous.

(b) If $T$ is positive and $E$ is $\sigma$-order continuous, then $T$ is $\sigma$-order continuous.

(c) If $T$ is positive and $E$ is order continuous, then $T$ is order continuous.

Proof. Suppose that $E$ is $\sigma$-order continuous and let $0 \leq e_{n} \uparrow e$ in $E$. Then $e_{n} \rightarrow e$ in norm of $E$. If $T$ is continuous then $T\left(e_{n}\right) \rightarrow T(e)$ in norm of $F$ and so (a) holds. If $T$ is positive (in particular, continuous), then $0 \leq T\left(e_{n}\right) \uparrow$ in $F$ and so $T\left(e_{n}\right) \uparrow T(e)$ in $F$, see [18, Theorem 100.4.(i)]. Therefore, (b) holds.

Now suppose that $E$ is order continuous and let $0 \leq e_{\tau} \uparrow e$ in $E$. Then we have that $\left\|e-e_{\tau}\right\|_{E} \downarrow 0$. If $T$ is positive then $0 \leq T\left(e_{\tau}\right) \uparrow$ in $F$ and $T\left(e_{\tau}\right) \leq T(e)$ for all $\tau$. Moreover, since $T$ is continuous, there exists a constant $K>0$ such that $\| T(e)-$ $T\left(e_{\tau}\right)\left\|_{F} \leq K\right\| e-e_{\tau} \|_{E}$ for all $\tau$. From all this, it follows that $T\left(e_{\tau}\right) \rightarrow T(e)$ in norm and so $T\left(e_{\tau}\right) \uparrow T(e)$, see [18, Theorem 100.8]. Therefore, (c) holds.

2.2. Banach function spaces. Given a measure space $(\Omega, \Sigma, \mu)$ without $\sigma$ finiteness assumptions on $\mu$, we denote by $L^{0}(\mu)$ the space of all measurable real functions on $\Omega$, where functions which are equal $\mu$-a.e. are identified.

By a B.f.s. $X(\mu)$ we mean a Banach space contained in $L^{0}(\mu)$ with norm $\|\cdot\|$ satisfying that if $g \in L^{0}(\mu)$ and $f \in X(\mu)$ with $|g| \leq|f| \mu$-a.e., then $g \in X(\mu)$ and $\|g\| \leq\|f\|$. In particular, $X(\mu)$ is a Banach lattice with the $\mu$-a.e. pointwise order. Note that the convergence in norm of a sequence implies the $\mu$-a.e. pointwise convergence for some subsequence. Also note that $0 \leq f_{n} \uparrow f$ in $X(\mu)$ if and only if $0 \leq f_{n} \uparrow f \mu$-a.e., that is, the lattice supremum coincides with the $\mu$-a.e. pointwise supremum. In general, this does not hold for upwards directed systems. From [13, Theorem 23.2.(ii)], it is direct to check that $X(\mu)$ is Dedekind $\sigma$-complete. Since $X(\mu)_{a}$ is an ideal in $X(\mu)$, it follows that $X(\mu)_{a}$ is also a Dedekind $\sigma$-complete B.f.s. and so $X(\mu)_{a n}=X(\mu)_{a}$. Then a B.f.s. is order continuous if and only if it is $\sigma$-order continuous. A weak unit $g \in X(\mu)$ is just a function $g>0 \mu$-a.e. From now and on we will simply write $f \leq g$ for $f \leq g$ $\mu$-a.e.

Lemma 2.2. Let $Y(\mu)$ be an ideal of a B.f.s. $X(\mu)$. The following statements are equivalent:

(a) $Y(\mu)$ has a weak unit. 
(b) $\Omega=\cup_{n} A_{n}$ with $A_{n} \in \Sigma$ such that $\chi_{A_{n}} \in Y(\mu)$.

(c) $X(\mu)$ has a weak unit and $Y(\mu)$ is super order dense in $X(\mu)$.

Proof. (a) $\Rightarrow$ (b). Take a weak unit $g \in Y(\mu)$ and a $\mu$-null set $Z$ such that $g(\omega)>0$ for all $\omega \in \Omega \backslash Z$. Then, for $B_{n}=\left\{\omega \in \Omega: g(\omega) \geq \frac{1}{n}\right\}$, it follows that $\Omega=\cup_{n} B_{n} \cup Z$. Note that $\chi_{B_{n}} \in Y(\mu)$ as $\chi_{B_{n}} \leq n g \in Y(\mu)$ and $\chi_{Z} \in Y(\mu)$ as $Z$ is $\mu$-null.

(b) $\Rightarrow$ (c). The function $g=\sum_{n} \frac{\chi_{A_{n}}}{2^{n}\left(\left\|\chi_{A_{n}}\right\|+1\right)}$ is a weak unit in $Y(\mu)$ and so in $X(\mu)$. On the other hand, for $0 \leq f \in X(\mu)$ and $D_{n}=\left(\cup_{j=1}^{n} A_{j}\right) \cap\{\omega \in \Omega: f(\omega) \leq n\}$, it follows that $0 \leq f \chi_{D_{n}} \uparrow f$ in $X(\mu)$ with $f \chi_{D_{n}} \in Y(\mu)$, as $0 \leq f \chi_{D_{n}} \leq n \chi_{\cup_{j=1}^{n}} A_{j} \in Y(\mu)$. That is, $Y(\mu)$ is super order dense in $X(\mu)$.

(c) $\Rightarrow$ (a). Take a weak unit $g \in X(\mu)$ and a $\mu$-null set $Z$ such that $g(\omega)>0$ for all $\omega \in \Omega \backslash Z$. Since $Y(\mu)$ is super order dense in $X(\mu)$, there exist $\left(f_{n}\right) \subset Y(\mu)$ and another $\mu$-null set $N$ such that $0 \leq f_{n}(\omega) \uparrow g(\omega)$ for all $\omega \in \Omega \backslash N$. Then, for $f=\sum_{n} \frac{f_{n}}{2^{n}\left(\left\|f_{n}\right\|+1\right)} \in$ $Y(\mu)$, it follows that the set $\{\omega \in \Omega: f(\omega) \leq 0\}$ is $\mu$-null as it is contained in $N \cup Z$. Hence, $f$ is a weak unit of $Y(\mu)$.

2.3. Integration with respect to a vector measure defined on a $\delta$-ring. Let $\Omega$ be an abstract set and $\mathcal{R}$ be a $\delta$-ring of subsets of $\Omega$ (i.e. a ring closed under countable intersections). Denote by $\mathcal{R}^{\text {loc }}$ the $\sigma$-algebra of all subsets $A$ of $\Omega$ such that $A \cap B \in \mathcal{R}$ for all $B \in \mathcal{R}$.

Consider a countably additive measure $\lambda: \mathcal{R} \rightarrow \mathbb{R}$, that is, $\sum \lambda\left(A_{n}\right)$ converges to $\lambda\left(\cup A_{n}\right)$ whenever $\left(A_{n}\right)$ is a sequence of pairwise disjoint sets in $\mathcal{R}$ with $\cup A_{n} \in \mathcal{R}$. The variation of $\lambda$ is the countably additive measure $|\lambda|: \mathcal{R}^{\text {loc }} \rightarrow[0, \infty]$ given by

$$
|\lambda|(A)=\sup \left\{\sum\left|\lambda\left(A_{i}\right)\right|:\left(A_{i}\right) \text { finite disjoint sequence in } \mathcal{R} \cap 2^{A}\right\} .
$$

The space $L^{1}(\lambda)$ of integrable functions with respect to $\lambda$ is defined as the space $L^{1}(|\lambda|)$ with the usual norm. Every $\mathcal{R}$-simple function $\varphi=\sum_{i=1}^{n} \alpha_{i} \chi_{A_{i}}$ (with $\left(A_{i}\right) \subset \mathcal{R}$ and $\left.\left(\alpha_{i}\right) \subset \mathbb{R}\right)$ is in $L^{1}(\lambda)$ and the integral of $\varphi$ with respect to $\lambda$ is defined by $\int \varphi d \lambda=$ $\sum_{i=1}^{n} \alpha_{i} \lambda\left(A_{i}\right)$. The $\mathcal{R}$-simple functions are dense in $L^{1}(\lambda)$. So the integral of $f \in L^{1}(\lambda)$ with respect to $\lambda$ is defined as $\int f d \lambda=\lim \int \varphi_{n} d \lambda$ for any sequence $\left(\varphi_{n}\right)$ of $\mathcal{R}$-simple functions converging to $f$ in $L^{1}(\lambda)$.

Let $E$ be a real Banach space and $v: \mathcal{R} \rightarrow E$ a vector measure, that is, $\sum v\left(A_{n}\right)$ converges to $v\left(\cup A_{n}\right)$ in $E$ whenever $\left(A_{n}\right)$ is a sequence of pairwise disjoint sets in $\mathcal{R}$ with $\cup A_{n} \in \mathcal{R}$. For every $e^{*}$ in the topological dual $E^{*}$ of $E$, the composition of $v$ with $e^{*}$ is a real measure denoted by $e^{*} v: \mathcal{R} \rightarrow \mathbb{R}$. The semi-variation of $v$ is the map $\|v\|: \mathcal{R}^{\text {loc }} \rightarrow[0, \infty]$ given by $\|v\|(A)=\sup \left\{\left|e^{*} \nu\right|(A): e^{*} \in B_{E^{*}}\right\}$, where $B_{E^{*}}$ is the unit ball of $E^{*}$. The semi-variation of $v$ is finite on $\mathcal{R}$ and satisfies

$$
\frac{\|v\|(A)}{2} \leq \sup \left\{\|v(B)\|_{E}: B \in \mathcal{R} \cap 2^{A}\right\} \leq\|v\|(A)
$$

for all $A \in \mathcal{R}^{\text {loc }}$. A set $A \in \mathcal{R}^{\text {loc }}$ is $\nu$-null if $v(B)=0$ for every $B \in \mathcal{R} \cap 2^{A}$ or equivalently $\|v\|(A)=0$. There always exists a measure $\lambda: \mathcal{R}^{\text {loc }} \rightarrow[0, \infty]$ with the same null sets as $\nu$, see [2, Theorem 3.2]. We will denote $L^{0}(v)=L^{0}(\lambda)$. A function $f \in L^{0}(v)$ is integrable with respect to $v$ if

(i) $f \in L^{1}\left(e^{*} v\right)$ for all $e^{*} \in E^{*}$, and 
(ii) for each $A \in \mathcal{R}^{\text {loc }}$ there exists a vector denoted by $\int_{A} f d \nu \in E$ such that

$$
e^{*}\left(\int_{A} f d \nu\right)=\int_{A} f d e^{*} v \text { for all } e^{*} \in E^{*}
$$

Let us denote by $L^{1}(v)$ the space of all integrable functions with respect to $v$ and by $L_{w}^{1}(v)$ the space of functions satisfying only condition (i). Both spaces are Banach function spaces related to $v$ (i.e. related to $\left(\Omega, \mathcal{R}^{\text {loc }}, \lambda\right)$ ) with the norm

$$
\|f\|_{\nu}=\sup _{e^{*} \in B_{E^{*}}} \int|f| d\left|e^{*} \nu\right| .
$$

Moreover, $L^{1}(v)$ is order continuous having the $\mathcal{R}$-simple functions as a dense subset and $L_{w}^{1}(v)$ has the $\sigma$-Fatou property. Note that it is not known if in general $L_{w}^{1}(v)$ has the Fatou property, see [3, Section 5]. Of course, $L^{1}(v) \subset L_{w}^{1}(v)$. The equality holds, for instance, if $E$ does not contain an isomorphic copy of $c_{0}$ (see [11, Theorem 5.1]), but there are plenty of cases where the containment is strict (see, for instance, the example in [3, Section 7] and also [3, Proposition 5.4]).

The integration operator $I_{v}: L^{1}(v) \rightarrow E$ given by $I_{v}(f)=\int_{\Omega} f d v$ is a continuous linear operator with $\left\|I_{\nu}(f)\right\|_{E} \leq\|f\|_{\nu}$ for all $f \in L^{1}(v)$.

For these and other issues related to integration with respect to a vector measure defined on a $\delta$-ring, see $[3,8,11,14,15]$.

3. First results. In this section we present some results that will be used later to prove our main theorems in Sections 4 and 5.

3.1. Results on Banach function spaces. Let $\mu$ and $\xi$ be two measures on a measurable space $(\Omega, \Sigma)$ such that $\xi \ll \mu$ (i.e. every $\mu$-null set is $\xi$-null). Then the map $[i]: L^{0}(\mu) \rightarrow L^{0}(\xi)$, which takes a $\mu$-a.e. class in $L^{0}(\mu)$ represented by $f$ into the $\xi$-a.e. class in $L^{0}(\xi)$ represented by the same $f$, is well defined. Consider two Banach function spaces, $X(\mu)$ and $Z(\xi)$. If $[i]: X(\mu) \rightarrow Z(\xi)$ is well defined then [i] is automatically continuous as it is positive. Note that $[i]$ is injective only in the case where $\mu$ and $\xi$ are equivalent, that is, they have the same null sets. In this case $L^{0}(\mu)=L^{0}(\xi)$ and $[i]$ is actually an inclusion, so it will be denoted by $i$.

Lemma 3.1. If $[i]: X(\mu) \rightarrow Z(\xi)$ then $[i]: X(\mu)_{a} \rightarrow Z(\xi)_{a}$.

Proof. Since [i]: $X(\mu) \rightarrow Z(\xi)$ is continuous, there exists a constant $K>0$ such that $\|f\|_{Z(\xi)} \leq K\|f\|_{X(\mu)}$ for all $f \in X(\mu)$. Let $f \in X(\mu)_{a}$. If $|f| \geq f_{n} \downarrow 0$ in $Z(\xi)$ or equivalently $\xi$-a.e., then there exists $A \in \Sigma$ such that $\Omega \backslash A$ is $\xi$-null and $|f| \geq f_{n} \chi_{A} \downarrow 0$ pointwise and so in $X(\mu)$. Hence, $\left\|f_{n}\right\|_{Z(\xi)}=\left\|f_{n} \chi_{A}\right\|_{Z(\xi)} \leq K\left\|f_{n} \chi_{A}\right\|_{X(\mu)} \downarrow 0$. That is, $f \in Z(\xi)_{a}$.

Lemma 3.2. Suppose that $X(\mu)_{a}$ is super order dense in $X(\mu)$ and $Z(\xi)$ has the $\sigma$-Fatou property. Then, if $[i]: X(\mu)_{a} \rightarrow Z(\xi)$, it follows that $[i]: X(\mu) \rightarrow Z(\xi)$.

Proof. Let $0 \leq f \in X(\mu)$ and take $\left(f_{n}\right) \subset X(\mu)_{a}$ such that $0 \leq f_{n} \uparrow f$ in $X(\mu)$ or equivalently $\mu$-a.e. and so $\xi$-a.e. Then $0 \leq f_{n} \uparrow$ in $Z(\xi)$ and $\sup \left\|f_{n}\right\|_{Z(\xi)} \leq$ $K \sup \left\|f_{n}\right\|_{X(\mu)} \leq K\|f\|_{X(\mu)}<\infty$ for some $K>0$. Since $Z(\xi)$ has the $\sigma$-Fatou property, there exists $g \in Z(\xi)$ such that $f_{n} \uparrow g$ in $Z(\xi)$ or equivalently $\xi$-a.e. Then, $f=g \xi$-a.e. 
and so $f \in Z(\xi)$. For a general $f \in X(\mu)$, by taking positive and negative parts of $f$, we obtain that $f \in Z(\xi)$.

Lemma 3.3. Suppose that $\mu$ and $\xi$ are equivalent, $X(\mu)_{a}$ is order dense in $L^{0}(\mu)$, and $Z(\xi)$ has the Fatou property. Then if $X(\mu)_{a} \subset Z(\xi)$, it follows that $X(\mu) \subset Z(\xi)$.

Proof. Let $0 \leq f \in X(\mu)$ and take $\left(f_{\tau}\right) \subset X(\mu)_{a}$ such that $0 \leq f_{\tau} \uparrow f$ in $L^{0}(\mu)$. Then $0 \leq f_{\tau} \uparrow$ in $Z(\xi)$ and $\sup \left\|f_{\tau}\right\|_{Z(\xi)} \leq K \sup \left\|f_{\tau}\right\|_{X(\mu)} \leq K\|f\|_{X(\mu)}<\infty$ for some $K>0$. Since $Z(\xi)$ has the Fatou property, there exists $g=\sup f_{\tau} \in Z(\xi)$. As $\mu$ and $\xi$ are equivalent and so $L^{0}(\mu)=L^{0}(\xi)$, we have that $f \leq g$ and thus $f \in Z(\xi)$, which, moreover, implies $f=g$.

3.2. Optimal extension for $\sigma$-order w-continuous operators. Fix a measurable space $(\Omega, \Sigma)$ to which every B.f.s. will be related. Let $X(\mu)$ be a B.f.s., $E$ a Banach space and $T: X(\mu) \rightarrow E$ a non-null linear operator. Consider the $\delta$-ring

$$
\mathcal{R}_{X(\mu)}=\left\{A \in \Sigma: \chi_{A} \in X(\mu)\right\}
$$

and the finitely additive set function $v_{T}: \mathcal{R}_{X(\mu)} \rightarrow E$ given by $v_{T}(A)=T\left(\chi_{A}\right)$. If $T$ is $\sigma$ order w-continuous, then $v_{T}$ is a vector measure as a consequence of the Orlicz-Pettis theorem, see, for instance, [10, Corollary I.4.4]. Note that $\Sigma \subset \mathcal{R}_{X(\mu)}^{\operatorname{loc}}$ and $v_{T} \ll \mu$ (i.e. every $\mu$-null set is $v_{T}$-null). The following result was proved in [4, Section 3].

THEOREM 3.4. If $T$ is $\sigma$-order $w$-continuous and $\Sigma=\mathcal{R}_{X(\mu)}^{\text {loc }}$, then the integration operator $I_{v_{T}}$ extends $T$ to $L^{1}\left(v_{T}\right)$, i.e.

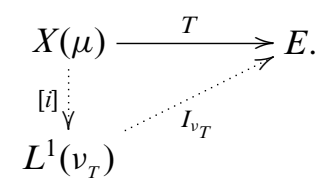

Moreover, the extension is optimal in the sense that if $Z(\xi)$ is a B.f.s. with $\xi \ll \mu$ such that

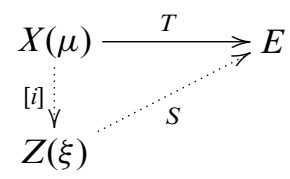

with $S$ being an $\sigma$-order $w$-continuous linear operator satisfying that for every $e^{*} \in E^{*}$ and $A \in \Sigma$ with $\chi_{A} \in Z(\xi)$,

$$
\sup _{B \in \mathcal{R}_{X(\mu)} \cap 2^{A}}\left|e^{*} S\left(\chi_{B}\right)\right|=0 \Rightarrow e^{*} S\left(\chi_{A}\right)=0,
$$

then $[i]: Z(\xi) \rightarrow L^{1}\left(v_{T}\right)$ is well defined and $S(f)=I_{v_{T}}(f)$ for all $f \in Z(\xi)$.

Note that under conditions of Theorem 3.4, since $I_{v_{T}}$ is continuous on $L^{1}\left(v_{T}\right)$ which is $\sigma$-order continuous, by Lemma 2.1.(a), $I_{v_{T}}$ is $\sigma$-order w-continuous. Moreover, since the $\mathcal{R}_{X(\mu)}$-simple functions are dense in $L^{1}\left(v_{T}\right)$, it follows that $I_{v_{T}}$ satisfies (3.1). The 
condition $\Sigma=\mathcal{R}_{X(\mu)}^{\text {loc }}$ is needed for the optimality to work, see [4, Lemma 2 and Proposition 4].

Let us consider the $\sigma$-finite case (i.e. $X(\mu)$ has a weak unit) and the discrete case (i.e. $\Sigma=2^{\Omega}$ and $\chi_{\{\omega\}} \in X(\mu)$ with $T\left(\chi_{\{\omega\}}\right) \neq 0$ for all $\left.\omega \in \Omega\right)$. In these two cases Theorem 3.4 can be written in a simpler way.

COROLlary 3.5. In the $\sigma$-finite or discrete case, if $T$ is $\sigma$-order $w$-continuous then $L^{1}\left(v_{T}\right)$ is the largest B.f.s. to which $T$ can be extended as a $\sigma$-order $w$-continuous operator still with values in $E$.

Proof. In the $\sigma$-finite case, by Lemma 2.2, there exists a sequence $\left(A_{n}\right) \subset \mathcal{R}_{X(\mu)}$ such that $\Omega=\cup A_{n}$. Then it is direct to check that $\Sigma=\mathcal{R}_{X(\mu)}^{\text {loc }}$ and condition (3.1) always holds for every $\sigma$-order w-continuous operator $S$. Note that, in this case, $v_{T}$ is a $\sigma$-finite vector measure, see [8, Section 3].

In the discrete case, $\Sigma=\mathcal{R}_{X(\mu)}^{\text {loc }}=2^{\Omega}$ and $v_{T}$ is a discrete vector measure, see [3, Lemma 4.6]. In particular, $v_{T}$ is equivalent to $\mu$ (the only null set for both is the empty set). Let us see that condition (3.1) always holds for every $\sigma$-order wcontinuous operator $S: Z(\xi) \rightarrow E$ extending $T$. Consider $v_{S}: \mathcal{R}_{Z(\xi)} \rightarrow E$ given by $v_{S}(A)=S\left(\chi_{A}\right)$, where $\mathcal{R}_{Z(\xi)}=\left\{A \in \Sigma: \chi_{A} \in Z(\xi)\right\}$. Since $S$ is $\sigma$-order w-continuous, $v_{S}$ is a vector measure. Moreover, for every $\omega \in \Omega$, since $\chi_{\{\omega\}} \in X(\mu)$ and $S$ extends $T$ to $Z(\xi)$, we have that $\{\omega\} \in \mathcal{R}_{Z(\xi)}$ and $v_{S}(\{\omega\})=S\left(\chi_{\{\omega\}}\right)=T\left(\chi_{\{\omega\}}\right) \neq 0$. That is, $\nu_{S}$ is discrete and so $\mathcal{R}_{Z(\xi)} \subset\{A \subset \Omega: A$ is countable $\}$. Then, given $A \in \mathcal{R}_{Z(\xi)}$, we can write $A=\cup_{n}\left\{\omega_{n}\right\}$. Since $\chi_{\cup_{j=1}^{n}\left\{\omega_{j}\right\}} \uparrow \chi_{A}$ in $Z(\xi)$ and $S$ is $\sigma$-order w-continuous, we have that $e^{*} S\left(\chi_{\cup_{j=1}^{n}\left\{\omega_{j}\right\}}\right) \rightarrow e^{*} S\left(\chi_{A}\right)$ for all $e^{*} \in E^{*}$, where $\cup_{j=1}^{n}\left\{\omega_{j}\right\} \in \mathcal{R}_{X(\mu)} \cap 2^{A}$. Hence, (3.1) holds. Note that $v_{T} \ll \xi$ as $[i]: Z(\xi) \rightarrow L^{1}\left(v_{T}\right)$ and so actually $\xi$ and $\mu$ are equivalent.

Under conditions of Theorem 3.4 we have that $T$ is continuous as it is the composition of two continuous operators. Since every continuous operator defined on a $\sigma$-order continuous Banach lattice is $\sigma$-order w-continuous (Lemma 2.1.(a)), then Corollary 3.5 gives the following result.

COROLlary 3.6. In the $\sigma$-finite or discrete case, if $T$ is $\sigma$-order $w$-continuous then $L^{1}\left(v_{T}\right)$ is the largest order continuous B.f.s. to which $T$ can be extended as a continuous operator still with values in $E$.

Recall that order continuity and $\sigma$-order continuity coincide for Banach function spaces. Let us remark that Corollaries 3.5 and 3.6 extend [6, Theorem 3.1 and Corollary 3.3] proved for the case where $\mu$ is finite and $X(\mu)$ is a B.f.s. in the sense of Lindenstrauss and Tzafriri [12].

3.3. Extension for the integration operator of a positive vector measure. Let $\mathcal{R}$ be a $\delta$-ring, $E$ be a Banach lattice and $v: \mathcal{R} \rightarrow E$ be a positive vector measure, that is, $v(A) \geq 0$ for all $A \in \mathcal{R}$. Then $I_{\nu}: L^{1}(v) \rightarrow E$ is a positive linear operator. Moreover, since $L^{1}(v)$ is order continuous, by Lemma 2.1.(c) we have that $I_{v}$ is order continuous. Note that the positivity of $v$ implies that $\|f\|_{v}=\left\|I_{v}(|f|)\right\|_{E}$ for all $f \in L^{1}(v)$. 
PROPOSITION 3.7. If E has the Fatou property, then there exists a positive order continuous linear operator $\bar{I}_{v}$ extending $I_{v}$ to $L_{w}^{1}(v)$, i.e.

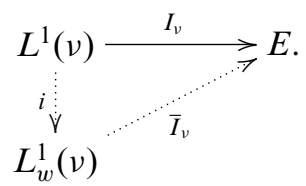

Proof. Let $0 \leq f \in L_{w}^{1}(v)$. By [3, Theorem 4.2] we have that $L^{1}(v)$ is order dense in $L_{w}^{1}(v)$, so there exists $\left(f_{\tau}\right) \subset L^{1}(v)$ such that $0 \leq f_{\tau} \uparrow f$ in $L_{w}^{1}(v)$. Then $0 \leq I_{\nu}\left(f_{\tau}\right) \uparrow$ in $E$, as $I_{\nu}$ is positive. Moreover, $\sup _{\tau}\left\|I_{\nu}\left(f_{\tau}\right)\right\|_{E}=\sup _{\tau}\left\|f_{\tau}\right\|_{\nu} \leq\|f\|_{\nu}<\infty$. So, since $E$ has the Fatou property, there exists $e=\sup _{\tau} I_{\nu}\left(f_{\tau}\right) \in E$. We define $\bar{I}_{v}(f):=e$. Note that $\bar{I}_{v}(f) \geq 0$ and $\bar{I}_{v}(f)=I_{v}(f)$ whenever $f \in L^{1}(v)$.

Let us see that $\bar{I}_{v}$ is well defined. Suppose that $\left(g_{\sigma}\right) \subset L^{1}(v)$ is such that $0 \leq$ $g_{\sigma} \uparrow f$ in $L_{w}^{1}(v)$ and denote $z=\sup _{\sigma} I_{\nu}\left(g_{\sigma}\right) \in E$. Then, for a fixed $\sigma$, we have that $f_{\tau} \wedge g_{\sigma} \uparrow_{\tau} f \wedge g_{\sigma}=g_{\sigma}$ in $L^{1}(v)$. Also, for a fixed $\tau$, we have that $f_{\tau} \wedge g_{\sigma} \uparrow_{\sigma} f_{\tau} \wedge f=f_{\tau}$ in $L^{1}(v)$. Since $I_{\nu}$ is order continuous, it follows that $I_{v}\left(g_{\sigma}\right)=\sup _{\tau} I_{\nu}\left(f_{\tau} \wedge g_{\sigma}\right)$ and $I_{\nu}\left(f_{\tau}\right)=\sup _{\sigma} I_{\nu}\left(f_{\tau} \wedge g_{\sigma}\right)$. Hence,

$$
I_{\nu}\left(f_{\tau} \wedge g_{\sigma}\right) \leq \sup _{\sigma} I_{\nu}\left(f_{\tau} \wedge g_{\sigma}\right)=I_{\nu}\left(f_{\tau}\right) \leq e
$$

for all $\tau$ and $\sigma$ and so $I_{\nu}\left(g_{\sigma}\right) \leq e$ for all $\sigma$. Then $z \leq e$. Similarly, $e \leq z$.

It is direct to check that $\bar{I}_{v}(f+g)=\bar{I}_{v}(f)+\bar{I}_{v}(g)$ for all $0 \leq f, g \in L_{w}^{1}(v)$. Then $\bar{I}_{v}$ has a unique positive linear extension $\bar{I}_{v}: L_{w}^{1}(v) \rightarrow E$, see, for instance, [18, Lemma 83.1]. If $f \in L^{1}(v)$, by taking positive and negative parts of $f$, we have $\bar{I}_{v}(f)=I_{v}(f)$.

Let us see now that $\bar{I}_{v}$ is order continuous. Suppose that $0 \leq f_{\tau} \uparrow f$ in $L_{w}^{1}(v)$. Then $0 \leq \bar{I}_{\nu}\left(f_{\tau}\right) \uparrow$ in $E$ with $\bar{I}_{\nu}\left(f_{\tau}\right) \leq \bar{I}_{\nu}(f)$ for all $\tau$. Since $E$ is Dedekind complete (as it has the Fatou property), there exits $\sup _{\tau} \bar{I}_{\nu}\left(f_{\tau}\right) \leq \bar{I}_{\nu}(f)$. Take $\left(g_{\sigma}\right) \subset L^{1}(v)$ such that $0 \leq g_{\sigma} \uparrow f$ in $L_{w}^{1}(v)$ and so $\bar{I}_{\nu}(f)=\sup _{\sigma} I_{\nu}\left(g_{\sigma}\right)$. For a fixed $\sigma$ we have $f_{\tau} \wedge g_{\sigma} \uparrow_{\tau} f \wedge g_{\sigma}=g_{\sigma}$ in $L^{1}(\nu)$ and then $I_{\nu}\left(g_{\sigma}\right)=\sup _{\tau} I_{\nu}\left(f_{\tau} \wedge g_{\sigma}\right)$. For every $\tau$ and $\sigma$, since $f_{\tau} \wedge g_{\sigma} \leq f_{\tau}$, it follows that

$$
I_{\nu}\left(f_{\tau} \wedge g_{\sigma}\right)=\bar{I}_{\nu}\left(f_{\tau} \wedge g_{\sigma}\right) \leq \bar{I}_{\nu}\left(f_{\tau}\right) \leq \sup _{\tau} \bar{I}_{\nu}\left(f_{\tau}\right)
$$

Hence, $I_{v}\left(g_{\sigma}\right) \leq \sup _{\tau} \bar{I}_{\nu}\left(f_{\tau}\right)$ for all $\sigma$ and so $\bar{I}_{v}(f) \leq \sup _{\tau} \bar{I}_{\nu}\left(f_{\tau}\right)$. Therefore, $\bar{I}_{v}\left(f_{\tau}\right) \uparrow$ $\bar{I}_{\nu}(f)$.

If we require $E$ to have only the $\sigma$-Fatou property, we can still extend $I_{\nu}$ but to a B.f.s. smaller than $L_{w}^{1}(v)$ and preserving the $\sigma$-order continuity of $I_{\nu}$. Denote by $\left[L^{1}(v)\right]_{\sigma-\mathrm{F}}$ the $\sigma$-Fatou completion of $L^{1}(v)$, that is, the minimal B.f.s. (related to $v$ ) with the $\sigma$-Fatou property containing $L^{1}(v)$. Note that $\left[L^{1}(v)\right]_{\sigma-\mathrm{F}}$ always exists, as $L_{w}^{1}(v)$ has the $\sigma$-Fatou property, and $L^{1}(v) \subset\left[L^{1}(v)\right]_{\sigma-\mathrm{F}} \subset L_{w}^{1}(v)$. Moreover, it follows that $L^{1}(v)$ is super order dense in $\left[L^{1}(v)\right]_{\sigma-\mathrm{F}}$, see $[3, \mathrm{p} .16]$. 
PROPOSITION 3.8. If E has the $\sigma$-Fatou property, then there exists a positive $\sigma$-order continuous linear operator $\bar{I}_{v}$ extending $I_{v}$ to $\left[L^{1}(v)\right]_{\sigma-\mathrm{F}}$, i.e.

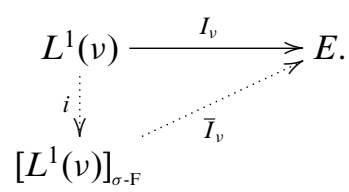

Proof. Let $0 \leq f \in\left[L^{1}(v)\right]_{\sigma-\mathrm{F}}$. Since $L^{1}(v)$ is super order dense in $\left[L^{1}(v)\right]_{\sigma-\mathrm{F}}$, there exists $\left(f_{n}\right) \subset L^{1}(v)$ such that $0 \leq f_{n} \uparrow f$ in $\left[L^{1}(v)\right]_{\sigma-\mathrm{F}}$. Then $0 \leq I_{v}\left(f_{n}\right) \uparrow$ in $E$ and $\sup _{n}\left\|I_{\nu}\left(f_{n}\right)\right\|_{E}=\sup _{n}\left\|f_{n}\right\|_{\nu} \leq\|f\|_{\nu}<\infty$. Since $E$ has the $\sigma$-Fatou property, there exists $e=\sup _{n} I_{v}\left(f_{n}\right) \in E$. We define $\bar{I}_{v}(f):=e$. Following the proof of Proposition 3.7 for sequences instead of nets, we obtain the conclusion.

4. Optimal extension for positive $\sigma$-order continuous operators. Fix a measurable space $(\Omega, \Sigma)$ to which every B.f.s. will be related. Let $X(\mu)$ be a B.f.s., $E$ be a Banach lattice with the $\sigma$-Fatou property and $T: X(\mu) \rightarrow E$ be a positive linear operator. Consider the order continuous part $X(\mu)_{a}$ of $X(\mu)$, the $\delta$-ring

$$
\mathcal{R}_{X(\mu)_{a}}=\left\{A \in \Sigma: \chi_{A} \in X(\mu)_{a}\right\}
$$

and the finitely additive set function $v_{T a}: \mathcal{R}_{X(\mu)_{a}} \rightarrow E$ given by $v_{T a}(A)=T\left(\chi_{A}\right)$. Since $T: X(\mu)_{a} \rightarrow E$ is continuous (as it is positive) on an order continuous B.f.s., by Lemma 2.1.(a), it is $\sigma$-order w-continuous. Then $v_{T a}$ is a vector measure (see Section 3.2). Note that $\Sigma \subset \mathcal{R}_{X(\mu)_{a}}^{\text {loc }}$ and $v_{T a} \ll \mu$. Since $v_{T a}$ is positive (as $T$ is so) and $E$ has the $\sigma$-Fatou property, we can consider the positive $\sigma$-order continuous linear operator $\bar{I}_{v_{T a}}$ given in Proposition 3.8 which extends $I_{v_{T a}}$ to $\left[L^{1}\left(v_{T a}\right)\right]_{\sigma-\mathrm{F}}$.

THEOREM 4.1. If $T$ is $\sigma$-order continuous, $\Sigma=\mathcal{R}_{X(\mu)_{a}}^{\text {loc }}$ and $X(\mu)_{a}$ is super order dense in $X(\mu)$, then the operator $\bar{I}_{v_{T a}}$ extends $T$ to $\left[L^{1}\left(v_{T a}\right)\right]_{\sigma-\mathrm{F}}$, i.e.

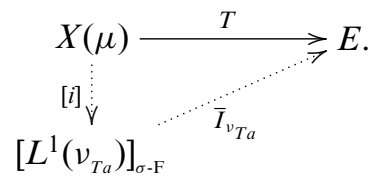

Moreover, the extension is optimal in the sense that if $Z(\xi)$ is a B.f.s. with $\xi \ll \mu$ such that $Z(\xi)_{a}$ is super order dense in $Z(\xi)$ and

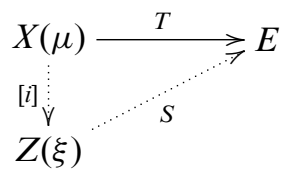

with $S$ being a positive $\sigma$-order continuous linear operator satisfying that for every $e^{*} \in E^{*}$ and $A \in \Sigma$ with $\chi_{A} \in Z(\xi)_{a}$,

$$
\sup _{B \in \mathcal{R}_{X(\mu) a} \cap 2^{A}}\left|e^{*} S\left(\chi_{B}\right)\right|=0 \Rightarrow e^{*} S\left(\chi_{A}\right)=0,
$$


then $[i]: Z(\xi) \rightarrow\left[L^{1}\left(v_{T a}\right)\right]_{\sigma \cdot \mathrm{F}}$ is well defined and $S(f)=\bar{I}_{v_{T a}}(f)$ for all $f \in Z(\xi)$.

Proof. Applying Theorem 3.4 to the restriction of $T$ to $X(\mu)_{a}$, we have that

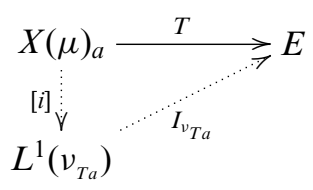

with the corresponding optimality. On the other hand, from Proposition 3.8,

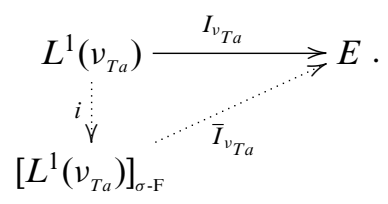

Then $[i]: X(\mu)_{a} \rightarrow\left[L^{1}\left(v_{T a}\right)\right]_{\sigma-\mathrm{F}}$ and so, by Lemma 3.2, $[i]: X(\mu) \rightarrow\left[L^{1}\left(v_{T a}\right)\right]_{\sigma . \mathrm{F}}$. Moreover, if $0 \leq f \in X(\mu)$, taking $\left(f_{n}\right) \subset X(\mu)_{a}$ such that $0 \leq f_{n} \uparrow f$ in $X(\mu)$, it follows that $0 \leq f_{n} \uparrow f$ in $\left[L^{1}\left(v_{T a}\right)\right]_{\sigma-\mathrm{F}}$ (see the proof of Lemma 3.2) with $\left(f_{n}\right) \subset L^{1}\left(v_{T a}\right)$. Since $\bar{I}_{v_{T a}}$ and $T$ are $\sigma$-order continuous, we have that

$$
\bar{I}_{v_{T a}}(f)=\sup \bar{I}_{v_{T a}}\left(f_{n}\right)=\sup I_{v_{T a}}\left(f_{n}\right)=\sup T\left(f_{n}\right)=T(f) .
$$

For a general $f \in X(\mu)$, by taking positive and negative parts of $f$, we also have that $\bar{I}_{v_{T a}}(f)=T(f)$. Therefore, (4.1) holds.

Let us see that the extension in (4.1) is optimal. Suppose $Z(\xi)$ is a B.f.s. with $\xi \ll \mu$ such that $Z(\xi)_{a}$ is super order dense in $Z(\xi)$ and (4.2) holds for a positive $\sigma$-order continuous linear operator $S$ satisfying (4.3). Since $[i]: X(\mu) \rightarrow Z(\xi)$, from Lemma 3.1, we have that $[i]: X(\mu)_{a} \rightarrow Z(\xi)_{a}$. Then,

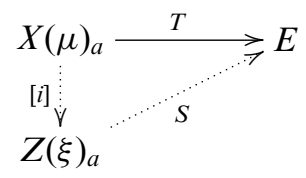

with $S: Z(\xi)_{a} \rightarrow E$ being $\sigma$-order w-continuous (Lemma 2.1.(a)) and satisfying (3.1) (as (4.3) holds). By the optimality of (4.4), we have that [i]: $Z(\xi)_{a} \rightarrow L^{1}\left(v_{T a}\right)$ and $S(f)=$ $I_{v_{T a}}(f)$ for all $f \in Z(\xi)_{a}$. Now, from Lemma 3.2, it follows that $[i]: Z(\xi) \rightarrow\left[L^{1}\left(v_{T a}\right)\right]_{\sigma-\mathrm{F}}$ and, since $S$ is $\sigma$-order continuous, it can be proved in the same way as for $T$ that $\bar{I}_{v_{T a}}(f)=S(f)$ for all $f \in Z(\xi)$.

Note that for a general vector measure $v$, we always have that $\left(\left[L^{1}(v)\right]_{\sigma-\mathrm{F}}\right)_{a}=L^{1}(v)$ is super order dense in $\left[L^{1}(v)\right]_{\sigma \cdot \mathrm{F}}$, see [3, p. 16]. Also note that, since the $\mathcal{R}_{X(\mu)_{a}}$-simple functions are dense in $L^{1}\left(v_{T a}\right)$, the operator $\bar{I}_{v_{T a}}$ satisfies (4.3). By Theorem 3.4, we have that $\bar{I}_{v_{T a}}$ is $\sigma$-order w-continuous only in the case where $\left[L^{1}\left(v_{T a}\right)\right]_{\sigma-\mathrm{F}}=L^{1}\left(v_{T a}\right)$.

In the $\sigma$-finite case, Theorem 4.1 gives the following result.

COROLLARY 4.2. Suppose that $X(\mu)_{a}$ has a weak unit. If $T$ is $\sigma$-order continuous, then $L_{w}^{1}\left(v_{T a}\right)$ is the largest B.f.s. to which $T$ can be extended as a positive $\sigma$-order continuous operator still with values in $E$. 
Proof. From Lemma 2.2, we can write $\Omega=\cup_{n} A_{n}$ with $\left(A_{n}\right) \subset \mathcal{R}_{X(\mu)_{a}}$. Then it is direct to check that $\Sigma=\mathcal{R}_{X(\mu)_{a}}^{\text {loc }}$. Moreover, $v_{T a}$ turns out to be $\sigma$-finite and so $\left[L^{1}\left(v_{T a}\right)\right]_{\sigma-\mathrm{F}}=L_{w}^{1}\left(v_{T a}\right)$, see [3, Theorem 5.1]. Also, by Lemma 2.2, we have that $X(\mu)_{a}$ is super order dense in $X(\mu)$.

On the other hand, if $[i]: X(\mu) \rightarrow Z(\xi)$, it follows that $Z(\xi)_{a}$ is super order dense in $Z(\xi)$. Indeed, by Lemma 3.1, we have that $[i]: X(\mu)_{a} \rightarrow Z(\xi)_{a}$ and so a weak unit in $X(\mu)_{a}$ is a weak unit in $Z(\xi)_{a}$. Then the conclusion follows from Lemma 2.2.

Finally, if $S$ is positive on $Z(\xi)$ and so it is $\sigma$-order w-continuous on $Z(\xi)_{a}$, for every $e^{*} \in E^{*}$ and $A \in \Sigma$ such that $\chi_{A} \in Z(\xi)_{a}$, it follows that $e^{*} S\left(\chi_{A}\right)=\lim _{n} e^{*} S\left(\chi_{A \cap\left(\cup_{j=1}^{n} A_{j}\right)}\right)$, and so (4.3) holds.

For the discrete case, Theorem 4.1 can also be written in a simpler way.

Corollary 4.3. Suppose that $\Sigma=2^{\Omega}$ and $\chi_{\{\omega\}} \in X(\mu)$ with $T\left(\chi_{\{\omega\}}\right) \neq 0$ for all $\omega \in \Omega$. If $T$ is $\sigma$-order continuous and $X(\mu)_{a}$ is super order dense in $X(\mu)$, then $\left[L^{1}\left(v_{T a}\right)\right]_{\sigma-\mathrm{F}}$ is the largest B.f.s. having its order continuous part as a super order dense subset to which $T$ can be extended as a positive $\sigma$-order continuous operator still with values in $E$.

Proof. Note that $\mu(\{\omega\})>0$ for all $\omega \in \Omega$ and so a set $A$ is $\mu$-null if and only if $A=$ $\emptyset$. Let us see that actually $\chi_{\{\omega\}} \in X(\mu)_{a}$ for all $\omega \in \Omega$. Suppose that $\chi_{\{\omega\}} \geq f_{n} \downarrow 0$ in $X(\mu)$ or equivalently pointwise. Then, $f_{n}=f_{n}(\omega) \chi_{\{\omega\}}$ and $\left\|f_{n}\right\|_{X(\mu)}=\left|f_{n}(\omega)\right| \cdot\left\|\chi_{\{\omega\}}\right\|_{X(\mu)} \downarrow 0$. Taking a look at the proof of Corollary 3.5 for $T: X(\mu)_{a} \rightarrow E$, we obtain the following conclusions. Firstly, $\Sigma=\mathcal{R}_{X(\mu)_{a}}^{\text {loc }}=2^{\Omega}$ and $v_{T a}$ is a discrete vector measure equivalent to $\mu$. Moreover, for every positive operator $S: Z(\xi) \rightarrow E$ extending $T$, since $S: Z(\xi)_{a} \rightarrow$ $E$ is $\sigma$-order w-continuous and extends $T: X(\mu)_{a} \rightarrow E$, we have that $S: Z(\xi)_{a} \rightarrow E$ satisfies (3.1), which is just condition (4.3) for $S$. Also note that $\xi$ is equivalent to $\mu$.

5. Optimal extension for positive order continuous operators. Consider the same conditions as in Section 4, but with $E$ having now the Fatou property. Our aim is to extend order continuous operators preserving the order continuity. The jump to nets requires extra conditions on the vector measure $v_{T a}$. Namely, assume that $\mathcal{R}_{X(\mu)_{a}}^{\text {loc }}=\Sigma$, the measures $v_{T a}$ and $\mu$ are equivalent and $L_{w}^{1}\left(v_{T a}\right)$ has the Fatou property. Let $\bar{I}_{v_{T a}}$ be the positive order continuous linear operator given in Proposition 3.7 which extends $I_{v_{T a}}$ to $L_{w}^{1}\left(v_{T a}\right)$.

THEOREM 5.1. If $T$ is order continuous and $X(\mu)_{a}$ is order dense in $L^{0}(\mu)$, then the operator $\bar{I}_{v_{T a}}$ extends $T$ to $L_{w}^{1}\left(v_{T a}\right)$, i.e.

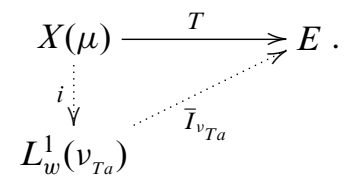

Moreover, the extension is optimal in the sense that if $Z(\xi)$ is a B.f.s. with $\xi \ll \mu$ such that $Z(\xi)_{a}$ is order dense in $L^{0}(\xi)$ and

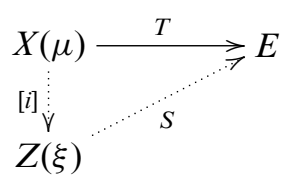


with $S$ being a positive order continuous linear operator satisfying that for every $e^{*} \in E^{*}$ and $A \in \Sigma$ with $\chi_{A} \in Z(\xi)_{a}$,

$$
\sup _{B \in \mathcal{R}_{X(\mu) a} \cap 2^{A}}\left|e^{*} S\left(\chi_{B}\right)\right|=0 \Rightarrow e^{*} S\left(\chi_{A}\right)=0,
$$

then $[i]: Z(\xi) \rightarrow L_{w}^{1}\left(v_{T a}\right)$ is well defined and $S(f)=\bar{I}_{v_{T a}}(f)$ for all $f \in Z(\xi)$.

Proof. Applying Theorem 3.4 to the restriction of $T$ to $X(\mu)_{a}$, we have

$$
\begin{aligned}
& X(\mu)_{a} \stackrel{T}{\longrightarrow} E \\
& \begin{array}{c}
{ }^{[i]} \\
L^{1}\left(v_{T a}\right)
\end{array}
\end{aligned}
$$

with the corresponding optimality. Actually, since $v_{T a}$ and $\mu$ are equivalent, $X(\mu)_{a} \subset$ $L^{1}\left(v_{T a}\right)$. On the other hand, by Proposition 3.7,

$$
\begin{aligned}
& L^{1}\left(v_{T a}\right) \stackrel{I_{v_{T a}}}{\longrightarrow} E . \\
& \begin{array}{c}
i_{w} \\
L_{w}^{1}\left(v_{T a}\right)
\end{array}
\end{aligned}
$$

Then $X(\mu)_{a} \subset L_{w}^{1}\left(v_{T a}\right)$ and so, by Lemma 3.3, $X(\mu) \subset L_{w}^{1}\left(v_{T a}\right)$. Moreover, if $0 \leq f \in$ $X(\mu)$, taking $\left(f_{\tau}\right) \subset X(\mu)_{a}$ such that $0 \leq f_{\tau} \uparrow f$ in $L^{0}(\mu)$, it follows that $0 \leq f_{\tau} \uparrow f$ in $L_{w}^{1}\left(v_{T a}\right)$ (see the proof of Lemma 3.3) with $\left(f_{\tau}\right) \subset L^{1}\left(v_{T a}\right)$. Since $\bar{I}_{v_{T a}}$ and $T$ are order continuous, we have that

$$
\bar{I}_{v_{T a}}(f)=\sup _{\tau} \bar{I}_{v_{T a}}\left(f_{\tau}\right)=\sup _{\tau} I_{v_{T a}}\left(f_{\tau}\right)=\sup _{\tau} T\left(f_{\tau}\right)=T(f) .
$$

For a general $f \in X(\mu)$, by taking positive and negative parts, we also have that $\bar{I}_{v_{T a}}(f)=T(f)$. So (5.1) holds.

Let us see that extension (5.1) is optimal. Suppose that $Z(\xi)$ is a B.f.s. with $\xi \ll \mu$ such that $Z(\xi)_{a}$ is order dense in $L^{0}(\xi)$ and (5.2) holds for a positive order continuous linear operator $S$ satisfying (5.3). Since $[i]: X(\mu) \rightarrow Z(\xi)$, from Lemma 3.1, we have that $[i]: X(\mu)_{a} \rightarrow Z(\xi)_{a}$. Then,

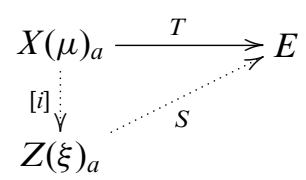

with $S$ being $\sigma$-order w-continuous (Lemma 2.1.(a)) and satisfying (3.1) (as (5.3) holds). By the optimality of (5.4), we have that $[i]: Z(\xi)_{a} \rightarrow L^{1}\left(v_{T a}\right)$ and $S(f)=I_{v_{T a}}(f)$ for all $f \in Z(\xi)_{a}$. Note that actually $\xi$ and $v_{T a}$ are equivalent as $v_{T a} \ll \xi \ll \mu$. From Lemma 3.3 , it follows that $Z(\xi) \subset L_{w}^{1}\left(v_{T a}\right)$ and, since $S$ is order continuous, it can be proved in the same way as for $T$ that $\bar{I}_{v_{T a}}(f)=S(f)$ for all $f \in Z(\xi)$.

Note that for a general vector measure $v$, we always have $\left(L_{w}^{1}(v)\right)_{a}=L^{1}(v)$ is order dense in $L^{0}(v)$, see [3, Theorem 3.2 and Remark 4.3]. Also note that $\bar{I}_{v_{T a}}$ satisfies (5.3) 
as the $\mathcal{R}_{X(\mu)_{a}}$-simple functions are dense in $L^{1}\left(v_{T a}\right)$. By Theorem 3.4, we have that $\bar{I}_{v_{T a}}$ is $\sigma$-order w-continuous only in the case where $L_{w}^{1}\left(v_{T a}\right)=L^{1}\left(v_{T a}\right)$.

In the $\sigma$-finite case (i.e. $X(\mu)_{a}$ has a weak unit), we always have that $\mathcal{R}_{X(\mu)_{a}}^{\text {loc }}=\Sigma$, $L_{w}^{1}\left(v_{T a}\right)$ has the Fatou property (see [3, Remark 5.5]) and $X(\mu)_{a}$ is order dense in $L^{0}(\mu)$ (see the proof of Lemma 2.2). Then, if we rewrite Theorem 5.1 for this case, we obtain that if $T$ is order continuous, then $L_{w}^{1}\left(v_{T a}\right)$ is the largest B.f.s. to which $T$ can be extended as a positive order continuous operator still with values in $E$. Note that since every order continuous operator is $\sigma$-order continuous, the same conclusion follows directly from Corollary 4.2 even if $E$ has only the $\sigma$-Fatou property and $v_{T a} \ll \mu$.

For the discrete case, Theorem 5.1 gives the following result.

COROLlary 5.2. Suppose that $\Sigma=2^{\Omega}$ and $\chi_{\{\omega\}} \in X(\mu)$ with $T\left(\chi_{\{\omega\}}\right) \neq 0$ for all $\omega \in \Omega$. If $T$ is order continuous, then $L_{w}^{1}\left(v_{T a}\right)$ is the largest B.f.s. to which $T$ can be extended as a positive order continuous operator still with values in $E$.

Proof. Note that $\Sigma=\mathcal{R}_{X(\mu)_{a}}^{\text {loc }}=2^{\Omega}$ and $v_{T a}$ is a discrete vector measure equivalent to $\mu$ (the only null set for both is the empty set), see the proof of Corollary 4.3. Moreover, $L_{w}^{1}\left(v_{T a}\right)$ has the Fatou property as $v_{T a}$ is discrete, see [3, Theorem 5.8].

On the other hand, $X(\mu)_{a}$ is order dense in $L^{0}(\mu)$. Indeed, let $0 \leq f \in L^{0}(\mu)$ and, for every finite set $I \subset \Omega$, consider $f \chi_{I}=\sum_{\omega \in I} f(\omega) \chi_{\{\omega\}} \in X(\mu)_{a}$. Then $0 \leq f \chi_{I} \uparrow f$ in $L^{0}(\mu)$. From the proof of Corollary 4.3, if $S: Z(\xi) \rightarrow E$ is a positive operator extending $T$, we have that $S$ satisfies (5.3) and $\xi$ is equivalent to $\mu$. Then, since $X(\mu)_{a} \subset Z(\xi)_{a} \subset L^{0}(\xi)=L^{0}(\mu)$, it follows that $Z(\xi)_{a}$ is order dense in $L^{0}(\xi)$.

6. Optimal extension for kernel operators. Firstly, let us point out some facts that will be used along this section. Let $v: \mathcal{R} \rightarrow E$ be a vector measure defined on a $\delta$-ring $\mathcal{R}$ and with values in a Banach space $E$.

LEMMA 6.1. The following assertions hold:

(a) If $\left(f_{n}\right) \subset L_{w}^{1}(v)$ is such that $0 \leq f_{n} \uparrow f v$-a.e., then $f \in L_{w}^{1}(v)$ if and only if $\sup _{n}\left\|f_{n}\right\|_{v}<\infty$.

(b) If $\left(f_{n}\right) \subset L^{1}(v)$ is such that $0 \leq f_{n} \uparrow f v$-a.e., then $f \in L^{1}(v)$ if and only if $\left(f_{n}\right)$ is a Cauchy sequence.

(c) If $\left(f_{n}\right) \subset L^{1}(v)$ is such that $0 \leq f_{n} \uparrow f$ v-a.e. with $f \in L_{w}^{1}(v)$, then $f \in L^{1}(v)$ if and only if $\lim _{n \rightarrow \infty}\left\|f-f_{n}\right\|_{v}=0$.

(d) If $E$ is an order continuous Banach lattice having the Fatou property, then $L^{1}(v)=\left[L^{1}(v)\right]_{\sigma-\mathrm{F}}=L_{w}^{1}(v)$.

(e) $\left[L^{1}(v)\right]_{\sigma-\mathrm{F}}=L_{w}^{1}(v)$ if and only if $v$ is locally $\sigma$-finite, that is, every $A \in \mathcal{R}^{\text {loc }}$ with $\|v\|(A)<\infty$ can be written as $A=\left(\cup A_{n}\right) \cup N$ with $N \in \mathcal{R}^{\text {loc }}$ being $v$-null and $\left(A_{n}\right) \subset \mathcal{R}$.

Proof. (a) Let $\left(f_{n}\right) \subset L_{w}^{1}(v)$ be such that $0 \leq f_{n} \uparrow f$-a.e. If $f \in L_{w}^{1}(v)$ then $\sup _{n}\left\|f_{n}\right\|_{\nu} \leq\|f\|_{\nu}<\infty$. Conversely, if $\sup _{n}\left\|f_{n}\right\|_{\nu}<\infty$, since $L_{w}^{1}(v)$ has the $\sigma$-Fatou property, we have that $f \in L_{w}^{1}(v)$.

(b) Let $\left(f_{n}\right) \subset L^{1}(v)$ be such that $0 \leq f_{n} \uparrow f v$-a.e. If $f \in L^{1}(v)$, since $L^{1}(v)$ is order continuous, we have that $f_{n} \rightarrow f$ in norm and so $\left(f_{n}\right)$ is a Cauchy sequence. Conversely, if $\left(f_{n}\right)$ is a Cauchy sequence, there exists $g \in L^{1}(v)$ such that $f_{n} \rightarrow g$ in norm. Then, as $L^{1}(v)$ is a B.f.s., there exists a subsequence $\left(f_{n_{k}}\right)$ such that $f_{n_{k}} \rightarrow g v$-a.e. So $f=g \in L^{1}(v)$. 
(c) Let $\left(f_{n}\right) \subset L^{1}(v)$ and $f \in L_{w}^{1}(v)$ be such that $0 \leq f_{n} \uparrow f v$-a.e. If $f \in L^{1}(v)$, since $L^{1}(v)$ is order continuous, we have that $f_{n} \rightarrow f$ in norm. The converse follows as $L^{1}(v)$ is closed in $L_{w}^{1}(v)$.

(d) We always have that $L^{1}(v) \subset\left[L^{1}(v)\right]_{\sigma-\mathrm{F}} \subset L_{w}^{1}(v)$. If $E$ is an order continuous Banach lattice having the Fatou property, then it does not contain an isomorphic copy of $c_{0}$ (see [1, Theorem 4.60 and Definition 4.58]) and so $L^{1}(v)=L_{w}^{1}(v)$ (see [11, Theorem 5.1]).

(e) This fact is proved in [3, Theorem 5.1].

Also, recall that if $E$ is a Banach lattice and $v$ is positive, then $\|f\|_{\nu}=\left\|I_{\nu}(|f|)\right\|_{E}$ for all $f \in L^{1}(v)$.

6.1. $\sigma$-finite case. Fix the measurable space $([0, \infty), \mathcal{B}[0, \infty))$, where $\mathcal{B}[0, \infty)$ is the $\sigma$-algebra of all Borel subsets of $[0, \infty)$, and denote by $m$ the Lebesgue measure on $[0, \infty)$. Let $K:[0, \infty) \times[0, \infty) \rightarrow[0, \infty)$ be a measurable function and consider the kernel operator $T$ defined by $K$ as

$$
T f(x)=\int_{0}^{\infty} f(y) K(x, y) d y
$$

for any measurable function $f$, for which the integral exists $m$-a.e. $x$.

Proposition 6.2. Let $1 \leq p, q \leq \infty$ and $p^{\prime}$ be the conjugate exponent of $p$. If

$$
K(x, \cdot) \in L^{p^{\prime}}(m) \text { for all } x \geq 0 \text { and } x \rightarrow\|K(x, \cdot)\|_{p^{\prime}} \in L^{q}(m),
$$

then $T: L^{p}(m) \rightarrow L^{q}(m)$ is well defined, linear, positive, $\sigma$-order continuous and

$$
\|T f\|_{q} \leq\|f\|_{p} \cdot\|\| K(x, \cdot)\left\|_{p^{\prime}}\right\|_{q} \text { for all } f \in L^{p}(m) .
$$

Proof. Given $f \in L^{p}(m)$, by Hölder's inequality, we have that

$$
\int_{0}^{\infty}|f(y)| K(x, y) d y \leq\|f\|_{p} \cdot\|K(x, \cdot)\|_{p^{\prime}}
$$

and so $T f \in L^{q}(m)$ with $\|T f\|_{q} \leq\|f\|_{p} \cdot\|\| K(x, \cdot)\left\|_{p^{\prime}}\right\|_{q}$. Hence, $T: L^{p}(m) \rightarrow L^{q}(m)$ is well defined and obviously linear and positive.

Let us see that $T$ is $\sigma$-order continuous. Recall that the lattice supremum of an increasing sequence in a B.f.s. coincides with the a.e. pointwise supremum. So, if $0 \leq f_{n} \uparrow f$ in $L^{p}(m)$, for every $x \geq 0$ we have that $0 \leq f_{n} K(x, \cdot) \uparrow f K(x, \cdot) m$-a.e. and, by the monotone convergence theorem,

$$
T f(x)=\int_{0}^{\infty} f(y) K(x, y) d y=\lim _{n \rightarrow \infty} \int_{0}^{\infty} f_{n}(y) K(x, y) d y=\lim _{n \rightarrow \infty} T f_{n}(x),
$$

that is $T f_{n} \uparrow T f$ in $L^{q}(m)$.

Assume that $K$ satisfies condition (6.1) and consider $T: L^{p}(m) \rightarrow L^{q}(m)$. Note that we are in the $\sigma$-finite case as $L^{p}(m)$ has a weak unit. In the cases $p<\infty$ or $p=\infty$ and $q<\infty$, we have that $T$ is $\sigma$-order w-continuous. The first case follows as $L^{p}(m)$ is order continuous. In the second one, if $0 \leq f_{n} \uparrow f m$-a.e., then $0 \leq T f_{n} \uparrow T f m$-a.e. 
(as $\mathrm{T}$ is $\sigma$-order continuous) and so $T f_{n} \rightarrow T f$ in norm (as $L^{q}(m)$ is order continuous). Therefore, by Corollaries 3.5 and 3.6, in both cases we have that

- $L^{1}\left(v_{T}\right)$ is the largest B.f.s. to which $T$ can be extended as a $\sigma$-order wcontinuous operator still with values in $L^{q}(m)$,

- $L^{1}\left(v_{T}\right)$ is the largest order continuous B.f.s. to which $T$ can be extended as a continuous operator still with values in $L^{q}(m)$,

where $v_{T}: \mathcal{R}_{L^{p}(m)} \rightarrow L^{q}(m)$ is the vector measure given by $v_{T}(A)=T\left(\chi_{A}\right)$. Note that $\mathcal{R}_{L^{p}(m)}=\{A \in \mathcal{B}[0, \infty): m(A)<\infty\}$ for $p<\infty$ and $\mathcal{R}_{L^{\infty}(m)}=\mathcal{B}[0, \infty)$. In the case $p=q=\infty$, the operator $T$ could not be $\sigma$-order w-continuous. For instance, if $T$ is the Hardy operator given by the kernel $K(x, y)=\frac{1}{x} \chi_{[0, x]}(y)$, then $T: L^{\infty}(m) \rightarrow L^{\infty}(m)$ is well defined but is not $\sigma$-order w-continuous. Indeed, in other case $v_{T}$ is a vector measure and so $\lim _{n \rightarrow \infty}\left\|v_{T}\left(\left(0, \frac{1}{n}\right]\right)\right\|_{\infty}=0$, but

$$
\left\|v_{T}\left(\left(0, \frac{1}{n}\right]\right)\right\|_{\infty}=\left\|T\left(\chi_{\left(0, \frac{1}{n}\right]}\right)\right\|_{\infty}=\sup _{x \geq 0} \frac{1}{x} m\left([0, x] \cap\left(0, \frac{1}{n}\right]\right)=1 .
$$

Note that Corollary 4.2 can be applied only for $p<\infty$ as $L^{\infty}(m)_{a}=\{0\}$. In this case, noting that $L^{q}(m)$ has the Fatou property, $v_{T a}=v_{T}$ as $L^{p}(m)_{a}=L^{p}(m)$ and $T$ is order continuous by Lemma 2.1.(c), we have that

- $L_{w}^{1}\left(v_{T}\right)$ is the largest B.f.s. to which $T$ can be extended as a positive $\sigma$-order continuous (and so also as order continuous) operator still with values in $L^{q}(m)$.

Consider a particular case where $T$ is the Hardy-type operator defined by the kernel $K(x, y)=\frac{1}{\phi(x)} \chi_{[0, x]}(y)$, with $\phi:[0, \infty) \rightarrow(0, \infty)$ being a measurable function such that $x^{1 / p^{\prime}} \phi(x)^{-1} \in L^{q}(m)$. This condition on $\phi$ guarantees that (6.1) holds for $K$ and so $T: L^{p}(m) \rightarrow L^{q}(m)$ looks as

$$
T f(x)=\frac{1}{\phi(x)} \int_{0}^{x} f(y) d y
$$

for all $f \in L^{p}(m)$. Different situations concerning the genuineness of the extensions for $T$ can occur.

(a) If $p<\infty, q<\infty$ and $x \phi(x)^{-1} \in L^{q}(m)$, then

$$
L^{p}(m) \varsubsetneqq L^{1}\left(v_{T}\right)=L_{w}^{1}\left(v_{T}\right) .
$$

(b) If $p<\infty, q=\infty$, $\phi$ is increasing, differentiable with increasing derivative and $\sum_{n \geq 1} \phi(n)^{-1}<\infty$, then

$$
L^{p}(m) \varsubsetneqq L^{1}\left(v_{T}\right) \varsubsetneqq L_{w}^{1}\left(v_{T}\right) .
$$

(c) If $p=1, q=\infty$ and $\phi(x)=1$ for all $x \geq 0$ (i.e. $T$ is the Volterra operator), then

$$
L^{1}(m)=L^{1}\left(v_{T}\right)=L_{w}^{1}\left(v_{T}\right)
$$


Under conditions of (a), for all $n \geq 1$, since $\chi_{[0, n]} \in L^{p}(m)$, we have

$$
\begin{aligned}
\left\|\chi_{[0, n]}\right\|_{v_{T}} & =\left\|I_{\nu_{T}}\left(\chi_{[0, n]}\right)\right\|_{q}=\left\|T\left(\chi_{[0, n]}\right)\right\|_{q} \\
& =\left(\int_{0}^{\infty} \frac{1}{\phi(x)^{q}}\left(\int_{0}^{x} \chi_{[0, n]}(y) d y\right)^{q} d x\right)^{1 / q} \\
& =\left(\int_{0}^{n} \frac{x^{q}}{\phi(x)^{q}} d x+\int_{n}^{\infty} \frac{n^{q}}{\phi(x)^{q}} d x\right)^{1 / q} \leq\left(\int_{0}^{\infty} \frac{x^{q}}{\phi(x)^{q}} d x\right)^{1 / q}
\end{aligned}
$$

and so $\sup _{n}\left\|\chi_{[0, n]}\right\|_{v_{T}}<\infty$. By Lemma 6.1.(a), $\chi_{[0, \infty)} \in L_{w}^{1}\left(v_{T}\right) \backslash L^{p}(m)$. Moreover, since $L^{q}(m)$ is order continuous and has the Fatou property, by Lemma 6.1.(d), $L^{1}\left(v_{T}\right)=$ $L_{w}^{1}\left(v_{T}\right)$.

Under conditions of (b), for every $n \geq 1$, since $\phi$ is increasing, it follows that

$$
\left\|\chi_{[n, n+1)}\right\|_{v_{T}}=\left\|T\left(\chi_{[n, n+1)}\right)\right\|_{\infty}=\sup _{x \geq 0} \frac{1}{\phi(x)} \int_{0}^{x} \chi_{[n, n+1)}(y) d y \leq \frac{1}{\phi(n)}
$$

and so $\left\|\chi_{[n, m)}\right\|_{v_{T}} \leq \sum_{j=n}^{m-1}\left\|\chi_{[j, j+1)}\right\|_{v_{T}} \leq \sum_{j=n}^{m-1} \phi(j)^{-1}$. Then $\left(\chi_{[0, n)}\right)$ is a Cauchy sequence in $L^{1}\left(v_{T}\right)$ and by Lemma 6.1.(b), $\chi_{[0, \infty)} \in L^{1}\left(v_{T}\right) \backslash L^{p}(m)$. On the other hand, noting that $0 \leq \phi^{\prime}(y) \chi_{[0, n]}(y) \leq \phi^{\prime}(n) \chi_{[0, n]}(y)$ for all $y \geq 0$, and so $\phi^{\prime} \chi_{[0, n]} \in L^{p}(m)$, we have

$$
\begin{aligned}
\left\|\phi^{\prime} \chi_{[0, n]}\right\|_{\nu_{T}} & =\left\|T\left(\phi^{\prime} \chi_{[0, n]}\right)\right\|_{\infty}=\sup _{x \geq 0} \frac{1}{\phi(x)} \int_{0}^{x} \phi^{\prime}(y) \chi_{[0, n]}(y) d y \\
& \leq \sup _{x \geq 0} \frac{1}{\phi(x)} \int_{0}^{x} \phi^{\prime}(y) d y=\sup _{x \geq 0} \frac{1}{\phi(x)}(\phi(x)-\phi(0)) \leq 1 .
\end{aligned}
$$

Then $\sup _{n}\left\|\phi^{\prime} \chi_{[0, n]}\right\|_{v_{T}}<\infty$ and so, by Lemma 6.1.(a), $\phi^{\prime} \in L_{w}^{1}\left(v_{T}\right)$. Moreover,

$$
\begin{aligned}
\left\|\phi^{\prime}-\phi^{\prime} \chi_{[0, n]}\right\|_{v_{T}} & \geq\left\|\phi^{\prime} \chi_{(n, m)}\right\|_{v_{T}}=\sup _{x \geq 0} \frac{1}{\phi(x)} \int_{0}^{x} \phi^{\prime}(y) \chi_{(n, m)}(y) d y \\
& \geq \sup _{n<x \leq m}\left(1-\frac{\phi(n)}{\phi(x)}\right)=\left(1-\frac{\phi(n)}{\phi(m)}\right)
\end{aligned}
$$

for all $m>n$ and noting that $\lim _{m \rightarrow \infty} \phi(m)^{-1}=0$ (as $\sum_{n \geq 1} \phi(n)^{-1}<\infty$ ), we have $\left\|\phi^{\prime}-\phi^{\prime} \chi_{[0, n]}\right\|_{v_{T}} \geq 1$ for all $n$. By Lemma 6.1.(c), $\phi^{\prime} \notin L^{1}\left(v_{T}\right)$.

Under conditions of (c), given $f \in L_{w}^{1}\left(v_{T}\right)$ and a sequence of simple functions $\left(\varphi_{n}\right)$ such that $0 \leq \varphi_{n} \uparrow|f|$, noting that

$$
\int_{0}^{k} \varphi_{n}(y) d y=\left\|T\left(\varphi_{n} \chi_{[0, k]}\right)\right\|_{\infty}=\left\|\varphi_{n} \chi_{[0, k]}\right\|_{v_{T}} \leq\|f\|_{v_{T}},
$$

by the monotone convergence theorem, we have

$$
\int_{0}^{\infty}|f(y)| d y=\lim _{n \rightarrow \infty} \lim _{k \rightarrow \infty} \int_{0}^{k} \varphi_{n}(y) d y \leq\|f\|_{v_{T}}
$$

and so $f \in L^{1}(m)$. 
REMARK 6.3. For instance, if $\phi(x)=e^{x}$ or $\phi(x)=(x+1)^{2+\alpha}$ with $\alpha>0$, then (a) and (b) hold.

6.2. Discrete case. Fix the measurable space $\left(\Gamma, 2^{\Gamma}\right)$, where $\Gamma$ is an uncountable set and denote by $\mu$ the counting measure on $\Gamma$. Given a map $K: \Gamma \times \Gamma \rightarrow[0, \infty)$, we consider the kernel operator $T$ defined by $K$ as

$$
T x=\left(\sum_{\gamma \in \Gamma} x_{\gamma} K(\sigma, \gamma)\right)_{\sigma \in \Gamma}
$$

for any family of real numbers $x=\left(x_{\gamma}\right)_{\gamma \in \Gamma}$, for which it is meaningful to do so.

Proposition 6.4. Let $1 \leq p, q \leq \infty$ and $p^{\prime}$ be the conjugate exponent of $p$. If

$$
K(\sigma, \cdot) \in \ell^{p^{\prime}}(\Gamma) \text { for all } \sigma \in \Gamma \text { and } \sigma \rightarrow\|K(\sigma, \cdot)\|_{p^{\prime}} \in \ell^{q}(\Gamma),
$$

then $T: \ell^{p}(\Gamma) \rightarrow \ell^{q}(\Gamma)$ is well defined, linear, positive, order continuous and

$$
\|T x\|_{q} \leq\|x\|_{p} \cdot\|\| K(\sigma, \cdot)\left\|_{p^{\prime}}\right\|_{q} \text { for all } x \in \ell^{p}(\Gamma) .
$$

Moreover, we are in the discrete case whenever

$$
K(\cdot, \gamma) \neq 0 \text { for all } \gamma \in \Gamma .
$$

Proof. Given $x=\left(x_{\gamma}\right)_{\gamma \in \Gamma} \in \ell^{p}(\Gamma)$, by the Hölder inequality, we have

$$
\sum_{\gamma \in \Gamma}\left|x_{\gamma}\right| K(\sigma, \gamma) \leq\|x\|_{p} \cdot\|K(\sigma, \cdot)\|_{p^{\prime}}
$$

and so $T x \in \ell^{q}(\Gamma)$ with $\|T x\|_{q} \leq\|x\|_{p} \cdot\|\| K(\sigma, \cdot)\left\|_{p^{\prime}}\right\|_{q}$. Hence, $T: \ell^{p}(\Gamma) \rightarrow \ell^{q}(\Gamma)$ is well defined and obviously linear and positive.

Let us see that $T$ is order continuous. Firstly, note that the lattice supremmum in $\ell^{p}(\Gamma)$ of an upwards directed system coincides with the pointwise supremmum. So, if $0 \leq x^{\tau} \uparrow x$ in $\ell^{p}(\Gamma)$, it follows that $0 \leq x^{\tau} K(\sigma, \cdot) \uparrow x K(\sigma, \cdot)$ in $\ell^{1}(\Gamma)$ for every $\sigma \in \Gamma$. Since $\ell^{1}(\Gamma)$ has the Fatou property, we have that

$$
T x^{\tau}(\sigma)=\left\|x^{\tau} K(\sigma, \cdot)\right\|_{1} \uparrow\|x K(\sigma, \cdot)\|_{1}=\operatorname{Tx}(\sigma)
$$

for every $\sigma \in \Gamma$ and so $T x^{\tau} \uparrow T x$ in $\ell^{q}(\Gamma)$.

Finally, note that $K(\cdot, \gamma) \neq 0$ is just $T\left(\chi_{\{\gamma\}}\right) \neq 0$ for all $\gamma \in \Gamma$, which defines the discrete case.

Assume that $K$ satisfies conditions (6.2) and (6.3) in Proposition 6.4 and consider $T: \ell^{p}(\Gamma) \rightarrow \ell^{q}(\Gamma)$. In the cases $p<\infty$ or $p=\infty$ and $q<\infty$, we have that $T$ is $\sigma$-order w-continuous. The first case follows as $\ell^{p}(\Gamma)$ is order continuous. In the second case, if $0 \leq x_{n} \uparrow x$ in $\ell^{\infty}(\Gamma)$, then $0 \leq T x_{n} \uparrow T x$ in $\ell^{q}(\Gamma)$ (as $T$ is order continuous) and so $T x_{n} \rightarrow T x$ in norm (as $\ell^{q}(\Gamma)$ is order continuous). Therefore, by Corollaries 3.5 and 3.6 , in both cases we have that

- $L^{1}\left(v_{T}\right)$ is the largest B.f.s. to which $T$ can be extended as a $\sigma$-order wcontinuous operator still with values in $\ell^{q}(\Gamma)$, 
- $L^{1}\left(v_{T}\right)$ is the largest order continuous B.f.s. to which $T$ can be extended as a continuous operator still with values in $\ell^{q}(\Gamma)$,

where $v_{T}: \mathcal{R}_{\ell^{p}(\Gamma)} \rightarrow \ell^{q}(\Gamma)$ is the vector measure given by $v_{T}(A)=T\left(\chi_{A}\right)$. Note that $\mathcal{R}_{\ell^{p}(\Gamma)}=\{A \subset \Gamma: A$ is finite $\}$ for $p<\infty$ and $\mathcal{R}_{\ell^{\infty}(\Gamma)}=2^{\Gamma}$. In the case where $p=\infty$ and $q=\infty$, the operator $T$ could not be $\sigma$-order w-continuous as we will see later.

In all cases $T$ is order continuous and $\ell^{q}(\Gamma)$ has the Fatou property, but $\ell^{p}(\Gamma)_{a}$ is super order dense in $\ell^{p}(\Gamma)$ only for $p<\infty$, in which case $\ell^{p}(\Gamma)_{a}=\ell^{p}(\Gamma)$ and $v_{T a}=v_{T}$. Note that $\ell^{\infty}(\Gamma)_{a}=c_{0}(\Gamma)$ is order dense but not super order dense in $\ell^{\infty}(\Gamma)$. Then, by Corollary 4.3 , for $p<\infty$ we have that

- $\left[L^{1}\left(v_{T}\right)\right]_{\sigma-\mathrm{F}}$ is the largest B.f.s. having its order continuous part as a super order dense subset, to which $T$ can be extended as a positive $\sigma$-order continuous operator still with values in $\ell^{q}(\Gamma)$.

Corollary 5.2 can be applied in all cases to obtain that

- $L_{w}^{1}\left(v_{T a}\right)$ is the largest B.f.s. to which $T$ can be extended as a positive order continuous operator still with values in $\ell^{q}(\Gamma)$,

where $v_{T a}$ is just $v_{T}$ for $p<\infty$ and the restriction of $v_{T}$ to $\mathcal{R}_{\ell^{\infty}(\Gamma)_{a}}=\mathcal{R}_{c_{0}(\Gamma)}=\{A \subset \Gamma$ : $A$ is finite for $p=\infty$.

Let us show that, as in the $\sigma$-finite case, the extensions can be or not genuine depending on the kernel.

Take $\Gamma=[0, \infty)$ and consider the Hardy-type operator $T$ defined by the kernel $K(\sigma, \gamma)=\phi(\sigma) \chi_{[0, \sigma]}(\gamma)$ with $\phi:[0, \infty) \rightarrow[0, \infty)$ satisfying that for every $\gamma \geq 0$ there exists $\sigma \geq \gamma$ such that $\phi(\sigma)>0$ (i.e. (6.3) holds for $K$ ). Assume $p=1$ and $\phi \in \ell^{q}(\Gamma)$ (the only way for (6.2) to hold) and so $T: \ell^{1}(\Gamma) \rightarrow \ell^{q}(\Gamma)$ looks as

$$
T x=\left(\phi(\sigma) \sum_{0 \leq \gamma \leq \sigma} x_{\gamma}\right)_{\sigma \geq 0}
$$

for all $x=\left(x_{\gamma}\right)_{\gamma \geq 0} \in \ell^{1}(\Gamma)$. Note that if $q<\infty$, since $\ell^{q}(\Gamma)$ is order continuous and has the Fatou property, by Lemma 6.1.(d), $L^{1}\left(v_{T}\right)=\left[L^{1}\left(v_{T}\right)\right]_{\sigma-\mathrm{F}}=L_{w}^{1}\left(v_{T}\right)$. Let us see that the second equality remains valid for $q=\infty$. We will write $\sharp(A)$ for the cardinal of set $A$. Suppose that there exist $A \subset[0, \infty)$ and $a>0$ such that $\sharp(A \cap[0, a])=\infty$ and consider $\left\{\gamma_{n}\right\}_{n \geq 1} \subset A \cap[0, a]$. Then, for all $n \geq 1$, we have

$$
\begin{aligned}
\left\|v_{T}\right\|(A) & \geq\left\|v_{T}\left(\cup_{j=1}^{n}\left\{\gamma_{j}\right\}\right)\right\|_{\infty}=\left\|T\left(\chi_{\cup_{j=1}^{n}\left\{\gamma_{j j}\right\}}\right)\right\|_{\infty} \\
& =\sup _{\sigma \geq 0} \phi(\sigma) \sharp\left([0, \sigma] \cap\left(\cup_{j=1}^{n}\left\{\gamma_{j}\right\}\right)\right) \geq n \cdot \sup _{\sigma \geq a} \phi(\sigma) \geq n \cdot \phi(b),
\end{aligned}
$$

where $b \geq a$ is such that $\phi(b)>0$, and so $\left\|v_{T}\right\|(A)=\infty$. Hence, every $A \subset[0, \infty)$ with $\left\|v_{T}\right\|(A)<\infty$ can be written as $A=\cup_{n \geq 1} A \cap[0, n]$ with $A \cap[0, n]$ finite, that is, $v_{T}$ is locally $\sigma$-finite, and so by Lemma 6.1.(e) $\left[L^{1}\left(v_{T}\right)\right]_{\sigma-\mathrm{F}}=L_{w}^{1}\left(v_{T}\right)$.

Let us show different situations:

(d) If $q<\infty$ and $(\sigma \phi(\sigma))_{\sigma \geq 0} \in \ell^{q}(\Gamma)$, then

$$
\ell^{1}(\Gamma) \varsubsetneqq L^{1}\left(v_{T}\right)=\left[L^{1}\left(v_{T}\right)\right]_{\sigma-\mathrm{F}}=L_{w}^{1}\left(v_{T}\right) .
$$

(e) If $q=\infty, \phi$ is decreasing and $\sum_{j \geq 1} \phi(j)<\infty$, then

$$
\ell^{1}(\Gamma) \varsubsetneqq L^{1}\left(v_{T}\right) \varsubsetneqq\left[L^{1}\left(v_{T}\right)\right]_{\sigma-\mathrm{F}}=L_{w}^{1}\left(v_{T}\right) .
$$


(f) If $q=\infty$ and $\phi(\sigma)=1$ for all $\sigma \geq 0$, then

$$
\ell^{1}(\Gamma)=L^{1}\left(v_{T}\right)=\left[L^{1}\left(v_{T}\right)\right]_{\sigma-\mathrm{F}}=L_{w}^{1}\left(v_{T}\right) .
$$

Under conditions of (d), for all $n \geq 1$, we have

$$
\begin{aligned}
\left\|\chi_{\cup_{j=1}^{n}\{j\}}\right\|_{\nu_{T}} & =\left\|I_{\nu_{T}}\left(\chi_{\cup_{j=1}^{n}\{j\}}\right)\right\|_{q}=\left\|T\left(\chi_{\cup_{j=1}^{n}\{j\}}\right)\right\|_{q} \\
& =\left(\sum_{\sigma \geq 0} \phi(\sigma)^{q} \sharp\left(\cup_{j=1}^{n}\{j\} \cap[0, \sigma]\right)^{q}\right)^{1 / q} \leq\left(\sum_{\sigma \geq 0} \phi(\sigma)^{q} \sigma^{q}\right)^{1 / q}
\end{aligned}
$$

and so $\sup _{n \geq 1}\left\|\chi_{\cup_{j=1}^{n}\{j\}}\right\|_{v_{T}}<\infty$. By Lemma 6.1.(a), $\chi_{\mathbb{N}} \in L_{w}^{1}\left(v_{T}\right) \backslash \ell^{1}(\Gamma)$.

Under conditions of (e), since

$$
\left\|\chi_{\{j\}}\right\|_{\nu_{T}}=\left\|T\left(\chi_{\{j\}}\right)\right\|_{\infty}=\sup _{\sigma \geq j} \phi(\sigma)=\phi(j),
$$

we have $\left\|\chi_{\cup_{j=n}^{m}\{j\}}\right\|_{v_{T}} \leq \sum_{j=n}^{m} \phi(j)$ and so $\left(\chi_{\cup_{j=1}^{n}\{j\}}\right)$ is a Cauchy sequence in $L^{1}\left(v_{T}\right)$. By Lemma 6.1.(b), $\chi_{\mathbb{N}} \in L^{1}\left(v_{T}\right) \backslash \ell^{1}(\Gamma)$. On the other hand, let $h=\left(h_{\gamma}\right)_{\gamma \geq 0}$ with $h_{\gamma}=$ $\left(\frac{1}{\phi(\gamma)}-\frac{1}{\phi(\gamma-1)}\right) \chi_{\mathbb{N}}(\gamma)$. Since

$$
\begin{aligned}
\left\|h \chi_{\cup_{j=1}^{n}\{j\}}\right\|_{\nu_{T}} & =\left\|T\left(h \chi_{\cup_{j=1}^{n}\{j\}}\right)\right\|_{\infty}=\sup _{\sigma \geq 0} \phi(\sigma) \sum_{\substack{j=1 \\
j \leq \sigma}}^{n} h_{j} \\
& \leq \sup _{\sigma \geq 0} \phi(\sigma)\left(\frac{1}{\phi(\sigma)}-\frac{1}{\phi(0)}\right) \leq 1
\end{aligned}
$$

and so $\sup _{n \geq 1}\left\|h \chi_{\cup_{j=1}^{n}\{j\}}\right\|_{v_{T}}<\infty$, by Lemma 6.1.(a), $h \in L_{w}^{1}\left(v_{T}\right)$. Moreover,

$$
\begin{aligned}
\left\|h-h \chi_{\cup_{j=1}^{n-1}\{j\}}\right\|_{\nu_{T}} & \geq\left\|h \chi_{\cup_{j=n}^{m}\{j\}}\right\|_{\nu_{T}}=\left\|T\left(h \chi_{\cup_{j=n}^{m}\{j\}}\right)\right\|_{\infty}=\sup _{\sigma \geq 0} \phi(\sigma) \sum_{\substack{j=n \\
j \leq \sigma}}^{m} h_{j} \\
& \geq \sup _{\sigma \in[n, m] \cap \mathbb{N}} \phi(\sigma)\left(\frac{1}{\phi(\sigma)}-\frac{1}{\phi(n-1)}\right)=1-\frac{\phi(m)}{\phi(n-1)}
\end{aligned}
$$

for all $m>n$. Then, since $\lim _{m \rightarrow \infty} \phi(m)=0$ as $\sum_{j \geq 1} \phi(j)<\infty$, we have that $\| h-$ $h \chi_{\cup_{j=1}^{n-1}\{j\}} \|_{v_{T}} \geq 1$ for all $n$ and so, by Lemma 6.1.(c), $h \notin L^{1}\left(v_{T}\right)$.

Under conditions of (f), if $f \in\left[L^{1}\left(v_{T}\right)\right]_{\sigma-\mathrm{F}}=L_{w}^{1}\left(v_{T}\right)$, from [3, Theorem 5.1], it follows that $\operatorname{supp}(f)=\left\{\gamma_{j}\right\}_{j \geq 1}$. Moreover,

$$
\sum_{j=1}^{n}\left|f\left(\gamma_{j}\right)\right|=\sup _{\sigma \geq 0} \sum_{\substack{j=1 \\ \gamma_{j} \leq \sigma}}^{n}\left|f\left(\gamma_{j}\right)\right|=\left\|T\left(|f| \chi_{\cup_{j=1}^{n}\left\{\gamma_{j}\right\}}\right)\right\|_{\infty}=\left\|f \chi_{\cup_{j=1}^{n}\left\{\gamma_{j}\right\}}\right\|_{\nu_{T}} \leq\|f\|_{\nu_{T}}
$$

and so $f \in \ell^{1}(\Gamma)$.

REMARK 6.5. For instance, (d) holds if $\phi(\sigma)=e^{-\sigma} \chi_{\mathbb{N}}(\sigma)$ or $\phi(\sigma)=\frac{1}{\sigma^{r}} \chi_{\mathbb{N}}(\sigma)$ with $r>1+\frac{1}{q}$, and (e) holds if $\phi(\sigma)=e^{-\sigma}$ or $\phi(\sigma)=\frac{1}{(\sigma+1)^{r}}$ with $r>1$.

Now consider the multiplier operator $T$ defined by $K(\sigma, \gamma)=\phi(\sigma) \chi_{\{\sigma\}}(\gamma)$ with $\phi:[0, \infty) \rightarrow(0, \infty)$. Note that (6.3) holds for $K$ as $\phi(\sigma)>0$ for all $\sigma \geq 0$. Assume 
$\phi \in \ell^{q}(\Gamma)$ (i.e. (6.2) holds) and so $T: \ell^{p}(\Gamma) \rightarrow \ell^{q}(\Gamma)$ looks as

$$
T x=\left(\phi(\sigma) x_{\sigma}\right)_{\sigma \geq 0}
$$

for all $x=\left(x_{\gamma}\right)_{\gamma \geq 0} \in \ell^{p}(\Gamma)$.

(g) If $p<\infty, q=\infty$ and $\lim _{n \rightarrow \infty} \phi(n)=0$, then

$$
\ell^{p}(\Gamma) \varsubsetneqq L^{1}\left(v_{T}\right) \varsubsetneqq\left[L^{1}\left(v_{T}\right)\right]_{\sigma-\mathrm{F}} \varsubsetneqq L_{w}^{1}\left(v_{T}\right) .
$$

(h) If $p=\infty, q=\infty$ and $(\sigma \phi(\sigma))_{\sigma \geq 0} \in \ell^{\infty}(\Gamma)$, then

$$
\ell^{\infty}(\Gamma) \varsubsetneqq L_{w}^{1}\left(v_{T a}\right) .
$$

Under conditions of $(\mathrm{g})$, since

$$
\left\|\chi_{\cup_{j=n}^{m}\{j\}}\right\|_{\nu_{T}}=\left\|T\left(\chi_{\cup_{j=n}^{m}\{j\}}\right)\right\|_{\infty}=\sup _{\sigma \in[n, m] \cap \mathbb{N}} \phi(\sigma),
$$

we have that $\left(\chi_{\cup_{j=1}^{n}\{j\}}\right)$ is a Cauchy sequence in $L^{1}\left(v_{T}\right)$ and so, by Lemma 6.1.(b), $\chi_{\mathbb{N}} \in L^{1}\left(v_{T}\right) \backslash \ell^{p}(\Gamma)$. On the other hand, taking $h=\frac{1}{\phi} \chi_{\mathbb{N}}$, we have

$$
\left\|h \chi_{\cup_{j=1}^{n}\{j\}}\right\|_{\nu_{T}}=\left\|T\left(h \chi_{\cup_{j=1}^{n}\{j\}}\right)\right\|_{\infty}=\sup _{\sigma \in[1, n] \cap \mathbb{N}} \phi(\sigma) h(\sigma)=1
$$

and so, by Lemma 6.1.(a), $h \in L_{w}^{1}\left(v_{T}\right)$. Since the support of $h$ is countable, it follows that $f \in\left[L^{1}\left(v_{T}\right)\right]_{\sigma-\mathrm{F}}($ see $[3$, Theorem 5.1]). Moreover,

$$
\left\|h \chi_{\cup_{j=n}^{m}\{j\}}\right\|_{\nu_{T}}=\left\|T\left(h \chi_{\cup_{j=n}^{m}\{j\}}\right)\right\|_{\infty}=\sup _{\sigma \in[n, m] \cap \mathbb{N}} \phi(\sigma) h(\sigma)=1
$$

and so, by Lemma 6.1.(b), $h \notin L^{1}\left(v_{T}\right)$. Finally, since

$$
\left\|v_{T}(A)\right\|_{\infty}=\left\|T\left(\chi_{A}\right)\right\|_{\infty}=\sup _{\sigma \in A} \phi(\sigma) \leq\|\phi\|_{\infty}
$$

for every $A \subset[0, \infty)$ finite, we have that

$$
\left\|\chi_{[0, \infty)}\right\|_{v_{T}}=\left\|v_{T}\right\|([0, \infty)) \leq 2 \sup \left\{\left\|v_{T}(A)\right\|_{\infty}: A \subset[0, \infty) \text { finite }\right\} \leq 2\|\phi\|_{\infty}<\infty
$$

and so $\chi_{[0, \infty)} \in L_{w}^{1}\left(v_{T}\right)$. Note that $\chi_{[0, \infty)} \notin\left[L^{1}\left(v_{T}\right)\right]_{\sigma-\mathrm{F}}$ as $[0, \infty)$ is not countable.

Under conditions of $(\mathrm{h})$, taking $h=\left(\gamma \chi_{\mathbb{N}}(\gamma)\right)_{\gamma \geq 0}$, since

$$
\left\|h \chi_{\cup_{j=1}^{n}\{j\}}\right\|_{v_{T a}}=\left\|T\left(h \chi_{\cup_{j=1}^{n}\{j\}}\right)\right\|_{\infty}=\sup _{\sigma \in[1, n] \cap \mathbb{N}} \sigma \phi(\sigma) \leq \sup _{\sigma \geq 0} \sigma \phi(\sigma),
$$

we have that $h \in L_{w}^{1}\left(v_{T a}\right) \backslash \ell^{\infty}(\Gamma)$.

REMARK 6.6. For instance, (g) and (h) hold if $\phi(\sigma)=e^{-\sigma}$ or $\phi(\sigma)=\frac{1}{\sigma+1}$. For these two functions, $T: \ell^{\infty}(\Gamma) \rightarrow \ell^{\infty}(\Gamma)$ is not $\sigma$-order w-continuous. In the other case, $v_{T}: 2^{\Gamma} \rightarrow \ell^{\infty}(\Gamma)$ is a vector measure and so $\lim _{n \rightarrow \infty}\left\|v_{T}\left(\cup_{j \geq n}\left\{\frac{1}{j}\right\}\right)\right\|_{\infty}=0$, but

$$
\left\|v_{T}\left(\cup_{j \geq n}\left\{\frac{1}{j}\right\}\right)\right\|_{\infty}=\left\|T\left(\chi_{\cup_{j \geq n}\left\{\frac{1}{j}\right\}}\right)\right\|_{\infty}=\sup _{j \geq n} \phi\left(\frac{1}{j}\right)=1 .
$$


ACKNOWLEDGEMENTS. The research was partially supported by MTM2012-36732C03-03 (Ministerio de Economía y Competitividad), FQM-262, FQM-4643, FQM7276 (Junta de Andalucía) and Feder Funds (European Union).

\section{REFERENCES}

1. C. D. Aliprantis and O. Burkinshaw, Positive operators (Academic Press, New York, NY, 1985).

2. J. K. Brooks and N. Dinculeanu, Strong additivity, absolute continuity and compactness in spaces of measures, J. Math. Anal. Appl. 45 (1974), 156-175.

3. J. M. Calabuig, O. Delgado, M. A. Juan and E. A. Sánchez Pérez, On the Banach lattice structure of $L_{w}^{1}$ of a vector measure on a $\delta$-ring, Collect. Math. (accepted) (to appear), doi: 10.1007/s13348-013-0081-8

4. J. M. Calabuig, O. Delgado and E. A. Sánchez Pérez, Factorizing operators on Banach function spaces through spaces of multiplication operators, J. Math. Anal. Appl. 364 (2010), $88-103$.

5. G. P. Curbera, Volterra convolution operators with values in rearrangement invariant spaces, J. London Math. Soc. 60 (1999), 258-268.

6. G. P. Curbera and W. J. Ricker, Optimal domains for kernel operators via interpolation, Math. Nachr. 244 (2002), 47-63.

7. G. P. Curbera and W. J. Ricker, Optimal domains for the kernel operator associated with Sobolev's inequality, Studia Math. 158 (2003), 131-152; Corregenda to Optimal domains for the kernel operator associated with Sobolev's inequality, Studia Math. 170 (2005), 217-218. $432-443$.

8. O. Delgado, $L^{1}$-spaces of vector measures defined on $\delta$-rings, Arch. Math. 84 (2005),

9. O. Delgado and J. Soria, Optimal domain for the Hardy operator, J. Funct. Anal. 244 (2007), 119-133.

10. J. Diestel and J. J. Uhl, Jr., Vector measures, American Mathematical Society Surveys, 15 (American Mathematical Society, Providence, RI, 1997).

11. D. R. Lewis, On integrability and summability in vector spaces, Illinois J. Math. 16 (1972), 294-307.

12. J. Lindenstrauss and L. Tzafriri, Classical Banach spaces, vol. II (Springer-Verlag, Berlin, Germany, 1979).

13. W. A. J. Luxemburg and A. C. Zaanen, Riesz spaces I (North-Holland, Amsterdam, Netherlands, 1971).

14. P. R. Masani and H. Niemi, The integration theory of Banach space valued measures and the Tonelli-Fubini theorems. I. Scalar-valued measures on $\delta$-rings, Adv. Math. 73 (1989), 204-241.

15. P. R. Masani and H. Niemi, The integration theory of Banach space valued measures and the Tonelli-Fubini theorems. II. Pettis integration, Adv. Math. 75 (1989), 121-167.

16. G. Mockenhaupt and W. J. Ricker, Optimal extension of the Hausdorff-Young inequality, J. Reine Angew. Math. 620 (2008), 195-211.

17. S. Okada and W. J. Ricker, Optimal domains and integral representations of convolution operators in $L^{p}(G)$, Integr. Equ. Oper. Theory 48 (2004), 525-546.

18. A. C. Zaanen, Riesz spaces II (North-Holland, Amsterdam, Netherlands, 1983). 